\title{
Magic coset decompositions
}

\author{
Sergio L. Cacciatori ${ }^{1,4}$, Bianca L. Cerchiai ${ }^{2,4}$, and \\ Alessio Marrani ${ }^{3}$
}

\author{
${ }^{1}$ Dipartimento di Scienze ed Alta Tecnologia, \\ Universit à degli Studi dell'Insubria, Via Valleggio 11, 22100 Como, Italy \\ sergio.cacciatori@uninsubria.it \\ ${ }^{2}$ Dipartimento di Matematica, \\ Università degli Studi di Milano, Via Saldini 50, 20133 Milano, Italy \\ bianca.cerchiai@unimi.it \\ ${ }^{3}$ Physics Department, Theory Unit, CERN, \\ CH 1211, Geneva 23, Switzerland \\ alessio.marrani@cern.ch \\ ${ }^{4}$ INFN, Sezione di Milano \\ Via Celoria, 16, 20133 Milano, Italy
}

\begin{abstract}
By exploiting a "mixed" non-symmetric Freudenthal-Rozenfeld-Tits magic square, two types of coset decompositions are analyzed for the non-compact special Kähler symmetric rank-3 coset $E_{7(-25)} /\left[\left(E_{6(-78)} \times\right.\right.$ $U(1)) / \mathbb{Z}_{3}$, occurring in supergravity as the vector multiplets' scalar manifold in $\mathcal{N}=2, D=4$ exceptional Maxwell-Einstein theory. The first decomposition exhibits maximal manifest covariance, whereas the second (triality-symmetric) one is of Iwasawa type, with maximal $S O(8)$ covariance. Generalizations to conformal non-compact, real forms of nondegenerate, simple groups "of type $E_{7}$ " are presented for both classes of coset parametrizations, and relations to rank-3 simple Euclidean Jordan algebras and normed trialities over division algebras are also discussed.
\end{abstract}

e-print archive: http://lanl.arXiv.org/abs/CERN-PH-TH/2012-020 


\section{Contents}

$\begin{array}{lll}1 & \text { Introduction } & 1078\end{array}$

2 The Lie algebra $\mathfrak{e}_{7(-25)}$ in the $56 \quad 1083$

2.1 The matrices $A_{\alpha}$ and the $d$-tensor of the 27 of $E_{6(-78)} \quad 1088$

$\begin{array}{lll}2.2 & \text { Properties of the matrices } Y_{A} & 1090\end{array}$

3 Manifestly $\left[\left(E_{6(-78)} \times U(1)\right) / \mathbb{Z}_{3}\right]$-covariant coset construction

$\begin{array}{lll}3.1 & \text { Remarks } & 1096\end{array}$

4 The Iwasawa decomposition 1099

$\begin{array}{llr}4.1 & S O(8)-\text { Triality } & 1102\end{array}$

4.2 Group theory 1104

4.2.1 A first chain of embeddings $\quad 1104$

4.2.2 A second chain of embeddings 1109

4.2.3 Comments on cartan subalgebras $\quad 1110$

5 Generalizations to groups of type $\mathrm{E}_{7} \quad 1112$

$\begin{array}{lll}6 & \text { Conclusion } & 1118\end{array}$

$\begin{array}{ll}\text { Acknowledgments } & 1121\end{array}$

$\begin{array}{ll}\text { References } & 1121\end{array}$

\section{Introduction}

The role of groups in Physics is at least 3-fold. First, they represent symmetries that, by definition, introduce elegance in all the equations which are manifestly symmetry invariant. If that was all, one may argue that this would be a poor advantage. But symmetries also arise as fundamental principles in constructing new theories, like, for example, gauge symmetries for the 
Standard Model of particle physics, conformal symmetry for string theory, or general covariance for the Einstein theory of relativity. Finally, symmetries, and then groups, play a key role in solving the equations of motion.

A particular class is represented by the (semi)simple Lie groups (and corresponding Lie algebras), which, once more, find application in a large number of mathematical and physical fields. All the finite dimensional complex Lie algebras have been classified by Wilhelm Killing, whose proofs have been made rigorous by Élie Cartan, who has also extended the classification to the non-compact, real cases. The well known result is that this classification has led to the discovery, beyond the famous classical series, of five exceptional algebras (of course together with the corresponding real forms): $\mathfrak{g}_{2}, \mathfrak{f}_{4}, \mathfrak{e}_{6}, \mathfrak{e}_{7}$, and $\mathfrak{e}_{8}$.

Despite their sporadicity, the appearance of exceptional Lie groups (and algebras) in physics is anything but sporadic [1]. The importance of compact exceptional Lie groups in realizing grand unification gauge theories and consistent string theories is well recognized. Similarly, the relevance of noncompact real forms for the study of locally supersymmetric theories of gravity is well known. Other examples include sigma models based on quotients of exceptional Lie groups, which are of interest for string theory and conformal field theory applications as well. It is worth mentioning that the analysis of quantum criticality in Ising chains and the structure of magnetic materials such as cobalt niobate has also recently (and strikingly) turned out to be related to exceptional Lie groups of type $E$ (see, e.g., [2,3], respectively).

Several properties of exceptional groups and algebras can be already inferred from abstract theoretical considerations. Nevertheless, it is often important to have explicit concrete realizations of the groups available in term of matrices, for both numerical or analytical calculations. For example, one could test conjectures related to confinement in non-Abelian gauge theories (see, e.g., [4]), and, more in general, perform explicit non-perturbative computations in exceptional lattice GUT theories and in random matrix theories.

In particular, among all the exceptional groups, there are specific motivations for physics to be interested in $E_{7}$ : recently, a strict relation between cryptography and black hole physics based on $E_{7}$ (and $E_{6}$ ) exceptional supergravity has been discovered [5-8]. However, actual computation of entangled expectation values requires again an explicit determination of the Haar measure and of the range of the parameters. Moreover, fascinating group theoretical structures arise clearly in the description of the Attractor Mechanism for black holes in the Maxwell-Einstein supergravity [9], such as the so-called magic exceptional supergravity [10] we are focusing on in the 


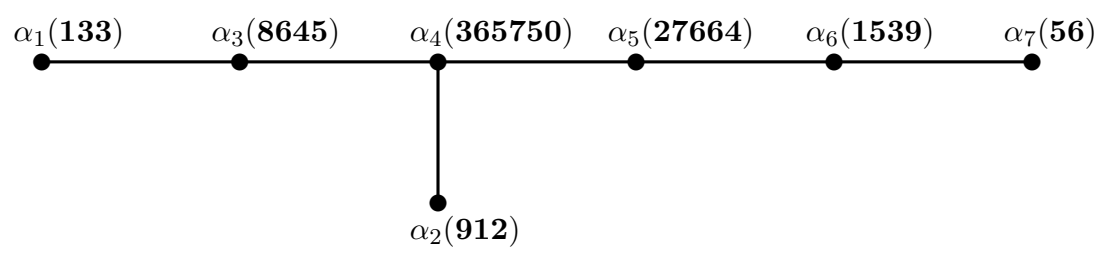

Figure 1: Dynkin diagram for $\mathfrak{e}_{7}$.

present investigation, which is related to the minimally non-compact real $E_{7(-25)}$ form [11] of $E_{7}$.

Before proceeding further, it is worth recalling some basic facts on the Lie algebra $\mathfrak{e}_{7}$ of $E_{7}$. Let us start by stating that $\mathfrak{e}_{7}$ is the unique exceptional Lie algebra of rank 7, and it is characterized by the Dynkin diagram drawn in figure 1 , in which each dot corresponds to a simple root $\alpha_{i}$. These are free generators of the root lattice $\Lambda_{R}=\sum_{i} \mathbb{Z} \alpha_{i}$. The space $H^{*}=\Lambda_{R} \otimes \mathbb{R}$ is endowed with a positive definite inner product $(\cdot \cdot \cdot)$. The weight lattice $\Lambda_{W}$ is the dual of $\Lambda_{R}$ with respect to the hooked product, which means that it is freely generated over $\mathbb{Z}$ by the fundamental weights $\lambda^{i} \in H^{*}, i=1, \ldots, 7$ defined by $\left\langle\alpha_{i}, \lambda^{j}\right\rangle=\delta_{i}^{j}$, with:

$$
\langle\alpha, \lambda\rangle:=2 \frac{(\alpha \mid \lambda)}{(\alpha \mid \alpha)} .
$$

There is a univocal correspondence between fundamental weights and fundamental representations, and all the irreducible finite dimensional representations can be generated from the basic ones, which are indicated in parenthesis in figure 1 . Here, we are going to deal with the two lower dimensional, namely the fundamental $\mathbf{5 6}$ and the adjoint $\mathbf{1 3 3}$.

The complex algebra $\mathfrak{e}_{7}$ is completely characterized by its Dynkin diagram, from which one can reconstruct the adjoint representation, that, being faithful, is isomorphic to the algebra itself. Since $\mathfrak{e}_{7}$ is a 133-dimensional complex algebra, it follows that such a representation is the aforementioned 133.

The Lie algebra $\mathfrak{e}_{7}$ exhibits four distinct non-compact, real forms. This means that there are four inequivalent ways to select a 133-dimensional real subspace of the 266-dimensional real space underlying the complex algebra $\mathfrak{e}_{7}$, in such a way that the selected subspace endowed with the inherited Lie product is itself a (real) Lie algebra. For each simple Lie algebra $\mathfrak{g}$ there is a unique simply connected Lie group $G$ (up to isomorphisms), such that 
Table 1: The real forms of $E_{7}$.

\begin{tabular}{lcc}
\hline Symbol & Real form & Maximal compact subgroup (mcs) \\
\hline$E_{7(-133)}$ & Compact & $E_{7(-133)}$ \\
$E_{7(7)}$ & Split & $S U(8) / \mathbb{Z}_{2}$ \\
$E_{7(-5)}$ & EVI & $(\operatorname{Spin}(12) \times U S p(2)) / \mathbb{Z}_{2}$ \\
$E_{7(-25)}$ & EVII & $\left(E_{6(-78)} \times U(1)\right) / \mathbb{Z}_{3}$ \\
\hline
\end{tabular}

$\mathfrak{g}$ is the corresponding Lie algebra. The complex Lie group $E_{7(\mathbb{C})}$ contains a maximal compact subgroup, which is a 133-dimensional real Lie group (denoted as $E_{7(-133)}$ ), whose Lie algebra is then called the compact form (denoted ${ }^{1}$ as $\mathfrak{e}_{7(-133)}$ ), where in parenthesis the signature of the Killing form (number of the positive eigenvalues minus number of the negative ones) is indicated.

The non-compact, real forms are in correspondence with the maximal compact subalgebras of $\mathfrak{e}_{7(-133)}$ (i.e., the compact Lie subalgebras that are not properly contained in a proper subalgebra of $\mathfrak{e}_{7(-133)}$ itself). The same holds at group level. There are four such subalgebras and therefore four corresponding real forms, which we collect in Table 1 (at Lie group level). For a recent treatment of $E_{7}$ groups (and algebras), see, e.g., [12].

The plan of the paper is as follows.

As anticipated, we are going to deal with the minimally non-compact real form of $\mathfrak{e}_{7}\left(E_{7}\right)$, namely with $\mathfrak{e}_{7(-25)}$ and its corresponding Lie group $E_{7(-25)}$, both denoted by EVII (see Table 1). In Section 2, by starting from its general construction through the Tits magic square, we study the Lie algebra $\mathfrak{e}_{7(-25)}$ itself, and we explicily construct a realization in the fundamental $\mathbf{5 6}$ representation embedded in $\mathfrak{u s p}(28,28)$. The matrix elements obtained with this technique turn out to be strictly related to the invariant totally symmetric rank-3 so-called $d$-tensor of the $E_{6(-78)}$ group, thus allowing for different expressions, depending on the choice of the basis for the relevant rank-3 (simple) Euclidean Jordan algebra.

\footnotetext{
${ }^{1}$ The Killing form $K$ on a complex Lie algebra is defined by $K(X, Y):=$ $\operatorname{Tr}(\operatorname{ad}(X) a d(Y))$ and is non-degenerate for a simple algebra and on the corresponding real forms. In particular, for a non-compact form it is negative definite on the maximal compact subalgebra, namely on the maximal Lie subalgebra, whose exponentiation generates a compact Lie (sub)group.
} 
In the present paper, we focus on two remarkable explicit parametrizations of the symmetric manifold ${ }^{2}$

$$
\mathcal{M}:=\frac{E_{7(-25)}}{K}=\frac{E_{7(-25)}}{\left(E_{6(-78)} \times U(1)\right) / \mathbb{Z}_{3}}
$$

(obtained by suitably exponentiating the corresponding coset Lie algebra), which can be regarded as the classical vector multiplets' scalar manifold of the $\mathcal{N}=2, D=4$ Maxwell-Einstein so-called exceptional magic supergravity theory, based on the rank-3 Euclidean simple Jordan algebra $\mathfrak{J}_{3}(\mathbb{O})$ on the normed division algebra of the octonions $\mathbb{O}[10]$.

The first type of coset parametrization/decomposition, analyzed in Section 3, exhibits maximal manifest covariance with respect to the maximal compact subgroup $(m c s) E_{6(-78)} \times U(1)$ of $E_{7(-25)}$ (up to $\mathbb{Z}_{3}$; see (1.2)). Interestingly, such a coset parametrization, also exhibiting a manifest complex (actually, special Kähler) structure, can be generalized to encompass a more general class of Lie groups, which in Section 5 we identify at least as the conformal non-compact real forms of simple, non-degenerate Lie groups "of type $E_{7}$ " [14], of which $E_{7(-25)}$ (in its $\mathbf{5 6}$ representation) can be considered as the generic representative. Groups "of type $E_{7}$ " have recently appeared in Theoretical Physics, in the investigation of single- [15] and multi-centered [16-20] extremal black hole solutions in supergravity theories, as well as in the study of matter creation in the Universe [21].

The second coset parametrization, studied in Section 4, relies on the Iwasawa construction, already analyzed for the split form $E_{7(7)}$ e.g., in [22]. In this case, the maximal manifest covariance reduces down to an $S O(8)$ subgroup of $E_{7(7)}$, which will interestingly turn out to be related to the automorphism group $\operatorname{Aut}(\mathbf{t}(\mathbb{O}))$ of the normed triality $\mathbf{t}(\mathbb{O})$ over the octonions (O) (entering the Tits' construction). The well known $S O(8)$ triality is manifest in such an approach, as detailed in the group theoretical analysis of Sections 4.1 and 4.2. As discussed in Section 5, also this construction of the Iwasawa decomposition can be generalized at least to the conformal non-compact real forms of simple, non-degenerate Lie groups "of type $E_{7}$ "; the resulting manifest covariance is then given by an $S O(q) \times \mathcal{A}_{q}$ subgroup, which remarkably shares the same Lie algebra as the automorphism group $\operatorname{Aut}(\mathbf{t}(\mathbb{A}))$ of the normed triality over the relevant normed division algebra (see, e.g., [23]) $\mathbb{A}=\mathbb{R}$ (reals), $\mathbb{C}$ (complex numbers), $\mathbb{H}$ (quaternions), and (1) (octonions).

\footnotetext{
${ }^{2}$ For previous studies of exceptional cosets in supergravity, see, e.g., [13].
} 
Table 2: The "mixed" magic square.

\begin{tabular}{lcccc}
\hline & $\mathbb{R}$ & $\mathbb{C}$ & $\mathbb{H}$ & $\mathbb{O}$ \\
\hline $\mathbb{R}$ & $S O(3)$ & $S U(3)$ & $U S p(6)$ & $F_{4(-52)}$ \\
$\mathbb{C}$ & $S U(3)$ & $S U(3) \times S U(3)$ & $S U(6)$ & $E_{6(-78)}$ \\
$\mathbb{H}_{S}$ & $S p(6, \mathbb{R})$ & $S U(3,3)$ & $S O^{*}(12)$ & $E_{7(-25)}$ \\
$\mathbb{O}_{S}$ & $F_{4(4)}$ & $E_{6(2)}$ & $E_{7(-5)}$ & $E_{8(-24)}$ \\
\hline
\end{tabular}

Final remarks, comments and discussion of further possible developments are given in the concluding Section 6 .

\section{The Lie algebra $\mathfrak{e}_{7(-25)}$ in the 56}

In order to construct the Lie algebra $\mathfrak{e}_{7(-25)}$, we are going to follow a procedure similar to the one outlined in Section 7 of Yokota [24], based on the non-symmetric "mixed" magic square $[10,25,26]$ displayed in Table 2.

The rows and the columns contain the division algebras of the real numbers $\mathbb{R}$, the complex numbers $\mathbb{C}$, the quaternions $\mathbb{H}$, and the octonions $\mathbb{O}$. Since at the group level we focus on the (minimally) non-compact form $E_{7(-25)}$, we need to start from the split form $\mathbb{H}_{S}$ of the quaternions in the third row. On the other hand, we are also interested in identifying explicitly its maximal compact subgroup $K:=E_{6(-78)} \times U(1) / \mathbb{Z}_{3}[24]$, and therefore the usual form $\mathbb{C}$ of the complex field in the second row is to be considered.

The Tits' formula then yields the Lie algebra $\mathcal{L}$ corresponding to division algebras in row $\mathbb{A}$ and column $\mathbb{B}$ as follows [26]:

$$
\mathcal{L}(\mathbb{A}, \mathbb{B})=\operatorname{Der}(\mathbb{A}) \oplus \operatorname{Der}\left(\mathfrak{J}_{3}(\mathbb{B})\right) \dot{+}\left(\mathbb{A}^{\prime} \otimes \mathfrak{J}_{3}^{\prime}(\mathbb{B})\right)
$$

The symbol $\oplus$ denotes the direct sum of algebras, whereas $\dot{+}$ stands for the direct sum of vector spaces. Moreover, Der are the linear derivations, $\mathfrak{J}_{3}(\mathbb{B})$ denotes the rank-3 Jordan algebra on $\mathbb{B}$, and the priming amounts to considering only traceless elements.

In particular, for the Lie algebra of $E_{7(-25)}$ the Tits' formula (2.1) reads:

$$
\mathfrak{e}_{7(-25)}=\mathcal{L}\left(\mathbb{H}_{S}, \mathbb{O}\right)=\operatorname{Der}\left(\mathbb{H}_{S}\right) \oplus \operatorname{Der}\left(\mathfrak{J}_{3}(\mathbb{O})\right) \dot{+}\left(\mathbb{H}_{S}^{\prime} \otimes \mathfrak{J}_{3}^{\prime}(\mathbb{O})\right)
$$


$\mathbb{H}_{S}^{\prime}$ denotes the imaginary split quaternions, and the following multiplication rule holds for the units $i, j, k \in \mathbb{H}_{S}$ (cf. e.g. (A.18) of [27]):

$i j=k=-j i, \quad j k=-i=-k j, \quad k i=j=-i k, \quad i^{2}=-1, \quad j^{2}=k^{2}=1$.

An inner product can be defined on $\mathbb{H}_{S}$ as:

$$
\left\langle h_{1}, h_{2}\right\rangle:=\operatorname{Re}\left(\bar{h}_{1} h_{2}\right), \quad h_{1}, h_{2} \in \mathbb{H}_{S} .
$$

Also, notice that if $L$ and $R$, respectively are the left and right translation in $\mathbb{H}_{S}$, then a derivation $D_{h_{1}, h_{2}} \in \operatorname{Der}\left(\mathbb{H}_{S}\right)$ can be constructed from $h_{1}$, $h_{2} \in \mathbb{H}_{S}$ as:

$$
D_{h_{1}, h_{2}}:=\left[L_{h_{1}}, L_{h_{2}}\right]+\left[R_{h_{1}}, R_{h_{2}}\right] .
$$

The rank-3 octonionic Jordan algebra $\mathfrak{J}_{3}(\mathbb{O})$ is defined as the algebra of the $3 \times 3$ Hermitian matrices of the form:

$$
J=\left(\begin{array}{ccc}
a_{1} & o_{1} & o_{2} \\
o_{1}^{*} & a_{2} & o_{3} \\
o_{2}^{*} & o_{3}^{*} & a_{3}
\end{array}\right)
$$

with $a_{i} \in \mathbb{R}$, and $o_{i} \in \mathbb{O}, i=1,2,3$. The Jordan product $\circ$ is thus realized as the symmetrized matrix multiplication:

$$
j_{1} \circ j_{2}:=\frac{1}{2}\left(j_{1} j_{2}+j_{2} j_{1}\right), \quad j_{1}, j_{2} \in \mathfrak{J}_{3}(\mathbb{O}) .
$$

It is then possible to introduce an inner product on the Jordan algebra:

$$
\left\langle j_{1}, j_{2}\right\rangle:=\operatorname{Tr}\left(j_{1} \circ j_{2}\right) .
$$

Furthermore, there is a cubic form, which is defined for any $j_{1}, j_{2}, j_{3} \in \mathfrak{J}_{3}(\mathbb{O})$ as $[28]$ (for a recent account, see, e.g., $[29,30]$ ):

$$
\begin{aligned}
\operatorname{Det}\left(j_{1}, j_{2}, j_{3}\right):= & \frac{1}{3} \operatorname{Tr}\left(j_{1} \circ j_{2} \circ j_{3}\right)-\frac{1}{6}\left(\operatorname{Tr}\left(j_{1}\right) \operatorname{Tr}\left(j_{2} \circ j_{3}\right)\right. \\
& \left.+\operatorname{Tr}\left(j_{2}\right) \operatorname{Tr}\left(j_{1} \circ j_{3}\right)+\operatorname{Tr}\left(j_{3}\right) \operatorname{Tr}\left(j_{1} \circ j_{2}\right)\right) \\
& +\frac{1}{6} \operatorname{Tr}\left(j_{1}\right) \operatorname{Tr}\left(j_{2}\right) \operatorname{Tr}\left(j_{3}\right) .
\end{aligned}
$$


In turn, this induces an action $\triangleright$ of $\mathfrak{J}_{3}(\mathbb{O})$ on itself through $\operatorname{Det}\left(j_{1}, j_{2}, j_{3}\right):=$ $\frac{1}{3} \operatorname{Tr}\left(\left(j_{1} \triangleright j_{2}\right) \circ j_{3}\right)$, which by definition (2.10) reads:

$$
\begin{aligned}
j_{1} \triangleright j_{2}:= & j_{1} \circ j_{2}-\frac{1}{2} \operatorname{Tr}\left(j_{1}\right) j_{2}-\frac{1}{2} \operatorname{Tr}\left(j_{2}\right) j_{1}+\frac{1}{2} \operatorname{Tr}\left(j_{1}\right) \operatorname{Tr}\left(j_{2}\right) I_{3} \\
& -\frac{1}{2} \operatorname{Tr}\left(j_{1} \circ j_{2}\right) I_{3},
\end{aligned}
$$

with $I_{3}$ the $3 \times 3$ identity matrix.

An important ingredient entering (2.1) is the Lie product $[\cdot, \cdot]$, which in the case under consideration extends the multiplication structure also to $\mathbb{H}_{S}^{\prime} \otimes \mathfrak{J}_{3}^{\prime}(\mathbb{O})$; its general explicit expression can be found, e.g., in (2.5) of [12]:

$$
\begin{aligned}
{\left[h_{1} \otimes j_{1}, h_{2} \otimes j_{2}\right]:=} & \frac{1}{12}\left\langle j_{1}, j_{2}\right\rangle D_{h_{1}, h_{2}}-\left\langle h_{1}, h_{2}\right\rangle\left[L_{j_{1}}, L_{j_{2}}\right] \\
& +\frac{1}{2}\left[h_{1}, h_{2}\right] \otimes\left(j_{1} \circ j_{2}-\frac{1}{3}\left\langle j_{1}, j_{2}\right\rangle I_{3}\right) .
\end{aligned}
$$

It is known (see, e.g., $[27,31])$ that:

$$
\begin{aligned}
\operatorname{Der}\left(\mathfrak{J}_{3}(\mathbb{O})\right) & \sim \mathfrak{f}_{4(-52)} ; \\
\operatorname{Der}\left(\mathbb{H}_{S}\right) & \sim \mathfrak{s l}(2, \mathbb{R}),
\end{aligned}
$$

and therefore $(2.2)$ can be recast as:

$$
\mathfrak{e}_{7(-25)}=\mathfrak{s l}(2, \mathbb{R}) \oplus \mathfrak{f}_{4} \dot{+}\left(\mathbb{H}_{S}^{\prime} \otimes \mathfrak{J}_{3}^{\prime}(\mathbb{O})\right),
$$

which implements the maximal non-symmetric embedding (whose compact form is given, e.g., by Table 15 of [32]; see also [33]):

$$
\begin{aligned}
E_{7(-25)} & \supset S L(2, \mathbb{R}) \times F_{4(-52)} ; \\
\mathbf{5 6} & =(\mathbf{4}, \mathbf{1})+(\mathbf{2}, \mathbf{2 6}) ; \\
\mathbf{1 3 3} & =(\mathbf{3}, \mathbf{1})+(\mathbf{1}, \mathbf{5 2})+(\mathbf{3}, \mathbf{2 6}) .
\end{aligned}
$$

We note in passing that, from the branching (2.15) of $\mathbf{5 6}$, this embedding is relevant for the maximal truncation of $\mathcal{N}=2, D=4$ magical exceptional theory (based on rank-3 simple Jordan algebra $\mathfrak{J}_{3}(\mathbb{O})$ ) to the smallest cubic $\mathcal{N}=2, D=4$ model, namely the so-called $T^{3}$ model, the truncation condition on the vectors (and their field strengths' fluxes, namely electric and magnetic charges) being given by $(\mathbf{2}, \mathbf{2 6})=0$.

As the next step, one needs to identify the subalgebra generating the maximal compact subgroup $K:=E_{6(-78)} \times U(1) / \mathbb{Z}_{3}$ of $E_{7(-25)}$. By considering 
the manifestly $\mathfrak{f}_{4(-52)}$-covariant decomposition of $\mathfrak{e}_{6(-78)}$ from Tits' formula (2.1):

$$
\mathfrak{e}_{6(-78)}=\mathcal{L}(\mathbb{C}, \mathbb{O})=\operatorname{Der}\left(\mathfrak{J}_{3}(\mathbb{O})\right) \dot{+}\left(i \otimes \mathfrak{J}_{3}^{\prime}(\mathbb{O})\right),
$$

the Lie algebra $\mathfrak{K}$ of $K$ can be identified as the subalgebra of $\mathfrak{e}_{6(-78)}$ defined by picking the only imaginary unit $i \in \mathbb{H}_{S}$ which satisfies $i^{2}=-1$ and computing:

$$
\mathfrak{K}=a d_{i} \oplus \operatorname{Der}\left(\mathfrak{J}_{3}(\mathbb{O})\right) \dot{+}\left(i \otimes \mathfrak{J}_{3}^{\prime}(\mathbb{O})\right),
$$

where $a d_{i} \in \mathbb{H}_{S}$ denotes the adjoint action of $i$, generating the maximal compact subgroup $U(1)$ of $S L(2, \mathbb{R})$. It is worth remarking that, due to the following property of the Lie product:

$$
\left[i \otimes j_{1}, i \otimes j_{2}\right]=-\left[L_{j_{1}}, L_{j_{2}}\right], \quad j_{1}, j_{2} \in \mathfrak{J}_{3}^{\prime}(\mathbb{O}),
$$

the multiplication of $\mathfrak{J}_{3}^{\prime}(\mathbb{O})$ by the imaginary unit $i$ in the last summand of (2.16) and (2.17) is exactly what is needed to get the compact form of $E_{6(-78)}$ instead of the (minimally) non-compact real form $E_{6(-26)}$, when exponentiating the algebra.

As anticipated, by this procedure, inspired by the approach of Cacciatori Piazza and Scotti [12] and exploiting the methods explained in [24], one can construct the (smallest symplectic) fundamental irrep. Fund $=\mathbf{5 6}$ of $E_{7(-25)}$ reproducing the structure constants of the $\mathbf{A d j}=\mathbf{1 3 3}$ irrep. (for whatever basis one chooses for the algebra).

Such an explicit symplectic realization reads as follows:

$$
\begin{aligned}
Y_{I} & =\left(\begin{array}{c|c|c|c}
\phi_{I} & \overrightarrow{0}_{27} & 0_{27} & \overrightarrow{0}_{27} \\
\hline \overrightarrow{0}_{27}^{T} & 0 & \overrightarrow{0}_{27}^{T} & 0 \\
\hline 0_{27} & \overrightarrow{0}_{27} & -\phi_{I}^{T} & \overrightarrow{0}_{27} \\
\hline \overrightarrow{0}_{27}^{T} & 0 & \overrightarrow{0}_{27}^{T} & 0
\end{array}\right), \quad I=1, \ldots, 78 ; \\
Y_{79} & =\left(\begin{array}{c|c|c|c}
\frac{i}{\sqrt{6}} I_{27} & \overrightarrow{0}_{27} & 0_{27} & \overrightarrow{0}_{27} \\
\hline \overrightarrow{0}_{27}^{T} & -i \sqrt{\frac{3}{2}} & \overrightarrow{0}_{27}^{T} & 0 \\
\hline 0_{27} & \overrightarrow{0}_{27} & -\frac{i}{\sqrt{6}} I_{27} & \overrightarrow{0}_{27} \\
\hline \overrightarrow{0}_{27}^{T} & 0 & \overrightarrow{0}_{27}^{T} & i \sqrt{\frac{3}{2}}
\end{array}\right) ;
\end{aligned}
$$




$$
\begin{aligned}
Y_{\alpha+79} & =\frac{1}{2}\left(\begin{array}{c|c|c|c}
0_{27} & \overrightarrow{0}_{27} & 2 i A_{\alpha} & i \sqrt{2} \vec{e}_{\alpha} \\
\hline \overrightarrow{0}_{27}^{T} & 0 & i \sqrt{2} \vec{e}_{\alpha}^{T} & 0 \\
\hline-2 i A_{\alpha} & -i \sqrt{2} \vec{e}_{\alpha} & 0_{27} & \overrightarrow{0}_{27} \\
\hline-i \sqrt{2} \vec{e}_{\alpha}^{T} & 0 & \overrightarrow{0}_{27}^{T} & 0
\end{array}\right), \quad \alpha=1, \ldots, 27 ; \\
Y_{\alpha+106} & =\frac{1}{2}\left(\begin{array}{c|c|c|c}
0_{27} & \overrightarrow{0}_{27} & -2 A_{\alpha} & \sqrt{2} \vec{e}_{\alpha} \\
\hline \overrightarrow{0}_{27}^{T} & 0 & \sqrt{2}_{2}^{T} & 0 \\
\hline-2 A_{\alpha} & \sqrt{2} \vec{e}_{\alpha} & 0_{27} & \overrightarrow{0}_{27} \\
\hline \sqrt{2} \vec{e}_{\alpha}^{T} & 0 & \overrightarrow{0}_{27}^{T} & 0
\end{array}\right), \quad \alpha=1, \ldots, 27,
\end{aligned}
$$

where $I_{n}$ is the $n \times n$ identity matrix, $0_{27}$ is the $27 \times 27$ null matrix, $\overrightarrow{0}_{n}$ is the zero vector in $\mathbb{R}^{n}$, and $\vec{e}_{\alpha}, \alpha=1, \ldots, 27$, is the canonical basis of $\mathbb{R}^{27}$ throughout.

The 78 matrices $\phi_{I}$ realize a subalgebra $\mathfrak{e}_{6(-78)}$ in its irreducible representation Fund $=27$. An explicit expression can be found, e.g., in Section 2.1 of $[12]$ :

$$
\phi_{I}= \begin{cases}C_{I} & I=1, \ldots, 52 \\ \tilde{C}_{I-52} & I=53, \ldots, 78\end{cases}
$$

where, in turn, the matrices $C_{I}$ realize a maximal $\mathfrak{f}_{4(-52)}$ subalgebra in its Fund $=\mathbf{2 6}$ irrep. (see, e.g., $[34,35])$.

The 27 matrices $A_{\alpha}$ are related to the $d$-tensor of $E_{6}$, as explained in more detail in Section 2.1 .

The first 79 matrices $Y_{I}(2.19)$ and $Y_{79}(2.20)$ generate the maximal compact subgroup $K$ of $E_{7(-25)}$ and are anti-Hermitian, whereas the remaining ones $Y_{\alpha+79}(2.21)$ and $Y_{\alpha+106}(2.22)$ generate the non-compact symmetric coset $E_{7(-25)} / K$ and they are Hermitian.

By introducing (cf. [12]):

$$
\widetilde{I}:=\left(\begin{array}{c|c}
I_{26} & \overrightarrow{0}_{26} \\
\hline \overrightarrow{0}_{26}^{T} & -2
\end{array}\right)
$$


the two matrices $Y_{106}$ and $Y_{133}$ can be rewritten more explicitly as:

$$
\begin{aligned}
Y_{106}= & \frac{1}{2}\left(\begin{array}{c|c|c|c}
0_{27} & \overrightarrow{0}_{27} & -i \sqrt{\frac{2}{3}} \widetilde{I} & i \sqrt{2} \vec{e}_{27} \\
\hline \overrightarrow{0}_{27}^{T} & 0 & i \sqrt{2} \vec{e}_{27}^{T} & 0 \\
\hline i \sqrt{\frac{2}{3}} \widetilde{I} & -i \sqrt{2} \vec{e}_{27} & 0_{27} & \overrightarrow{0}_{27} \\
\hline-i \sqrt{2} \vec{e}_{27}^{T} & 0 & \overrightarrow{0}_{27}^{T} & 0
\end{array}\right), \\
Y_{133}= & \frac{1}{2}\left(\begin{array}{c|c|c|c}
0_{27} & \overrightarrow{0}_{27} & \sqrt{\frac{2}{3}} \widetilde{I}^{2} & \sqrt{2}_{27} \\
\hline \overrightarrow{0}_{27}^{T} & 0 & \sqrt{2} \vec{e}_{27}^{T} & 0 \\
\hline \sqrt{\frac{2}{3}} \widetilde{I} & \sqrt{2} \vec{e}_{27} & 0_{27} & \overrightarrow{0}_{27} \\
\hline \sqrt{2} \vec{e}_{27}^{T} & 0 & \overrightarrow{0}_{27}^{T} & 0
\end{array}\right) .
\end{aligned}
$$

Together with $Y_{79}\left(U(1)\right.$ generator), $Y_{106}$ and $Y_{133}$ generate an $S L(2, \mathbb{R})$ subgroup, corresponding to the one appearing in (2.14) and (2.15).

\subsection{The matrices $A_{\alpha}$ and the $d$-tensor of the 27 of $E_{6(-78)}$}

By choosing a basis $\left\{j_{a}\right\}_{a=1, \ldots, 26}$ of $\mathfrak{J}_{3}^{\prime}(\mathbb{O})$ normalized as $\left\langle j_{a}, j_{b}\right\rangle=2 \delta_{a b}$, a completion to a basis for $\mathfrak{J}_{3}(\mathbb{O})$ can be obtained by adding $j_{27}=\sqrt{\frac{2}{3}} I_{3}$. The $A_{\alpha}$ 's are $27 \times 27$ symmetric matrices representing, by means of the linear isomorphism $\mathfrak{J}_{3}(\mathbb{O}) \simeq \mathbb{R}^{27}, \quad j_{\alpha} \mapsto \vec{e}_{\alpha}$, the action $\triangleright$ of $\mathfrak{J}_{3}(\mathbb{O})$ on $\mathfrak{J}_{3}(\mathbb{O})$ itself. The components of $A_{\alpha}$, explicitly computed in [12], satisfy the following relation [28]:

$$
\left(A_{\alpha}\right)_{\gamma}^{\beta}=\frac{1}{2} \operatorname{Tr}\left(\left(j_{\alpha} \triangleright j_{\gamma}\right) \circ j_{\beta}\right)=\frac{3}{2} \operatorname{Det}\left(j_{\alpha}, j_{\gamma}, j_{\beta}\right)=: \frac{1}{\sqrt{2}} d_{\alpha \gamma \beta},
$$

where $d_{\alpha \gamma \beta}=d_{(\alpha \gamma \beta)}$ is the totally symmetric rank-3 invariant $d$-tensor of the 27 of of $E_{6(-78)}$, with a normalization suitable to match $\operatorname{Det}\left(j_{\alpha}, j_{\gamma}, j_{\beta}\right)$ given by (2.10) (see below). We point out that the result (2.27) does not depend on the choice of the basis $\left\{j_{\alpha}\right\}$. Thus, the expressions of $Y_{\alpha+79}(2.21)$ and of $Y_{\alpha+106}(2.22)$ exhibit the maximal manifest compact $\left[\left(E_{6(-78)} \times U(1)\right) / \mathbb{Z}_{3}\right]$ covariance. However, whenever the choice of the basis $\left\{j_{\alpha}\right\}$ is exploited in order to distinguish the identity matrix from the traceless ones, the $d_{\alpha \beta \gamma}$ of $E_{6}$ has a maximal manifestly $F_{4(-52)}$-invariance only. This also holds for the expressions of the $Y_{I}(2.19)$, which are manifestly $F_{4(-52)^{-c o v a r i a n t}}$ only, due 
to the splitting (2.23). Notice that the full $\left[\left(E_{6(-78)} \times U(1)\right) / \mathbb{Z}_{3}\right]$-covariance can be recovered simply by picking a generic basis for the Jordan algebra.

A manifestly $[S U(6) \times S U(2)]$-invariant expression of the $d$-tensor of the 27 of $E_{6(-78)}$ has been constructed in [36]. On the other hand, $d$-tensors for the non-compact real forms of $E_{6}$ have been more extensively considered in the literature, e.g., due to their appearance in the general form of the holomorphic prepotential $F$ of cubic special Kähler geometry (see, e.g., [37]). For instance, in [38] the $d$-tensors of $E_{6(6)}$ (split) and $E_{6(-26)}$ (minimally non-compact) real forms have been explicitly constructed, with $U S p(8)$ and $U S p(6,2)$ maximal manifest invariance, respectively. By denoting with $G_{6}$ the $U$-duality ${ }^{3}$ group of chiral supergravity theories with 8 supersymmetries in $D=6$ space-time dimensions, and considering all $U$-duality groups $G_{5}$ of $\mathcal{N}=2, D=5$ supergravity theories with symmetric (vector multiplets') scalar manifold, manifestly $\left[G_{6} \times S O(1,1)\right]$-invariant expressions of the $G_{5}$-invariant $d$-tensor have been derived, e.g., in [37,41-45].

A necessary remark on the consistence of normalizations is in order. As a consequence of the choice (2.35) for the normalization of the matrices $Y_{A}$ discussed in Section 2.2, the components $\left(A_{\alpha}\right)^{\beta}{ }_{\gamma}:=A_{\alpha \beta \gamma}$ are normalized as:

$$
A_{\alpha \beta \gamma} A^{\eta \beta \gamma}=5 \delta_{\alpha}^{\eta}
$$

This is consistent with the normalization of the $d$-tensor (of $\left.E_{6(-26)}\right)$ given by the following expression of the Kähler-invariant $\left(\left(X^{0}\right)^{2}\right.$-rescaled) holomorphic prepotential function characterizing special Kähler geometry (see, e.g., $[37,46,47])$ :

$$
f(z):=\frac{1}{3 !} d_{\alpha \beta \gamma} z^{\alpha} z^{\beta} z^{\gamma}
$$

adopted, e.g., in [48]; in general, $\alpha=1, \ldots, n_{V}$, where $n_{V}$ denotes the number of Abelian vector multiplets coupled to the supergravity multiplet. Indeed, within the notation conventions adopted in [49], one can compute that (see also $[50,51])$ :

$$
d_{\alpha \beta \gamma} d^{\eta \beta \gamma}=(q+2) \delta_{\alpha}^{\eta}
$$

\footnotetext{
${ }^{3}$ Here $U$-duality is referred to as the "continuous" symmetries of Cremmer and Julia [39]. Their discrete versions are the $U$-duality non-perturbative string theory symmetries introduced by Hull and Townsend [40].
} 
For all the models reported in Table 3 below but the $T^{3}$ model, $q$ can be defined as:

$$
q \equiv \operatorname{dim}_{\mathbb{R}} \mathbb{A},
$$

where $\mathbb{A}$ denotes the division algebra on which the corresponding rank-3 simple Jordan algebra $\mathfrak{J}_{3}(\mathbb{A})$ is constructed $(q=8,4,2,1$ for $\mathbb{A}=\mathbb{O}, \mathbb{H}, \mathbb{C}$, $\mathbb{R}$, respectively). Furthermore, as observed in [52], in general $q$ is related to the inverse Coxeter number $\lambda$ by the relation:

$$
\begin{aligned}
& \lambda=-\frac{2}{q+2}, \quad q=0,1,2,4,8 \\
& \lambda=-\frac{1}{q+1}, \quad q=-2 / 3\left(T^{3} \text { model }\right) .
\end{aligned}
$$

The case $q=0$ in (2.32) corresponds to the triality symmetric so-called $\mathcal{N}=$ $2 S T U$ model [53], based on $\mathfrak{J}_{3}=\mathbb{R} \oplus \boldsymbol{\Gamma}_{1,1} \sim \mathbb{R} \oplus \mathbb{R} \oplus \mathbb{R}$; however, since the corresponding $U$-duality group $G_{4}$ is semi-simple, it will not be considered in the present investigation.

Coming back to the previous reasoning, by plugging $q=8$ (corresponding to the octonionic theory considered above) into (2.30), one achieves the following result:

$$
q=8: d_{\alpha \beta \gamma} d^{\eta \beta \gamma}=10 \delta_{\alpha}^{\eta}
$$

which matches (2.28) when taking (2.27) into account, and assuming for the $d$-tensor of $E_{6(-78)}$ the same normalization of the $d$-tensor of $E_{6(-26)}$.

\subsection{Properties of the matrices $Y_{A}$}

The $Y_{A}$ 's are orthonormalized (with signature $\left(-{ }^{79},+^{54}\right)$ ) with respect to the product:

$$
\left\langle Y, Y^{\prime}\right\rangle_{\mathbf{5 6}}:=\frac{1}{12} \operatorname{Tr}\left(Y Y^{\prime}\right)
$$

This normalization guarantees that the period of the maximal torus in the $E_{6}$ subgroup equals $4 \pi$, which is the standard choice for the period of the spin representations of the orthogonal subgroups [34,35].

Furthermore, the complete symmetry of the $d$-tensor implies the matrices $Y_{A}(A=1, \ldots, 133)$ given by the expressions $(2.19)-(2.22)$ to be symplectic 
with respect to the canonical symplectic form:

$$
\Omega:=\left(\begin{array}{cc}
0_{28} & -I_{28} \\
I_{28} & 0_{28}
\end{array}\right)
$$

namely (in a block-wise notation, and suppressing the index $A$ ):

$$
Y:=\left(\begin{array}{ll}
A & B \\
C & D
\end{array}\right) \in \mathfrak{s p}(56, \mathbb{C}) \Leftrightarrow \Omega Y+Y^{T} \Omega=0 \Leftrightarrow\left\{\begin{array}{l}
A^{T}=-D \\
B^{T}=B \\
C^{T}=C
\end{array} .\right.
$$

Actually, it holds that:

$$
Y \in \mathfrak{u s p}(28,28) .
$$

In order to show this, let us introduce:

$$
\mathcal{H}:=\left(\begin{array}{cc}
I_{28} & 0_{28} \\
0_{28} & -I_{28}
\end{array}\right),
$$

and recall the infinitesimal condition:

$$
Y \in \mathfrak{u}(28,28) \Leftrightarrow \mathcal{H} Y+Y^{\dagger} \mathcal{H}=0 \Leftrightarrow\left\{\begin{array}{l}
A^{\dagger}=-A \\
B^{\dagger}=C \\
D^{\dagger}=-D
\end{array} .\right.
$$

Thus, by means of the isomorphism:

$$
\mathfrak{s p}(2 n, \mathbb{R}) \sim \mathfrak{u} \mathfrak{s p}(n, n) \equiv \mathfrak{s p}(2 n, \mathbb{C}) \cap \mathfrak{u}(n, n),
$$

it follows that:

$$
Y \in \mathfrak{u s p}(28,28) \Leftrightarrow\left\{\begin{array}{c}
A=-A^{\dagger}=\bar{D} \\
C=B^{\dagger}=\bar{B} .
\end{array}\right.
$$

It should be noted that, when considering $n$ vector fields in presence of scalar fields, the isomorphism (2.41) has been exploited by Gaillard and Zumino [54] for the study of the generalization and non-compact nature of the electric-magnetic symmetry, naturally yielding in $D=4$ a manifestly $U S p(n, n)$-covariant basis of self-dual/anti-self-dual vector 2-form field strengths, rather than an $S p(2 n, \mathbb{R})$-covariant one; see also, e.g., the reelaboration of such a treatment presented in [47]. 
Let us analyze the properties of the matrices $Y_{A}(2.19)-(2.22)$ (following the notation of [12]):

(1) $Y_{I}$ (2.19) with $I=1, \ldots, 52$. According to $(2.23), \phi_{I}=C_{I}$. Up to a change of basis of the Jordan algebra, the matrices $C_{I}$ are given in [34] (including the Mathematica routine used for their computation). As mentioned before, the $C_{I}$ 's realize a maximal $\mathfrak{f}_{4(-52)}$ subalgebra in its irreducible representation Fund $=\mathbf{2 6}$. In turn, this is embedded into the algebra $\mathfrak{e}_{6(-78)}$ (maximal compact subalgebra of $\mathfrak{e}_{7(-25)}$ ) in its Fund $=\mathbf{2 7}$ irrep., through the addition of an extra 27th row and column of 0's, according to the maximal and symmetric embedding: $E_{6} \supset F_{4}, \mathbf{2 7}=\mathbf{2 6}+\mathbf{1}$. The symmetry properties are:

$$
C_{I}=-C_{I}^{T}, \quad \overline{C_{I}}=C_{I} \Longrightarrow Y_{I}=-Y_{I}^{\dagger} \in \mathfrak{u s p}(28,28) .
$$

(2) $Y_{I}$ (2.19) with $I=53, \ldots, 78$. According to $(2.23), \Phi_{I}=\tilde{C}_{I-52}$, as computed in [35], where the Mathematica routine to generate them is given, as well. The fact that the $\tilde{C}$ 's are purely imaginary is a consequence of the presence of the factor $i$ in the last summand of (2.16); they are defined in terms of the action (2.10) applied to the traceless part $\mathfrak{J}_{3}^{\prime}(\mathbb{O})$ of the Jordan algebra. In turn, such an action of the Jordan algebra on itself is the one entering the cubic form and hence in the definition (2.27) of the matrices $A_{\alpha}$ 's, implying that the $\tilde{C}_{I-52}$ coincide with the first 26 components of $A_{\alpha}$, apart from an overall $i$. The symmetry properties are:

$$
\tilde{C}_{I-52}=\tilde{C}_{I-52}^{T}, \quad \tilde{C}_{I-52}^{\dagger}=-\tilde{C}_{I-52} \Longrightarrow Y_{I}=-Y_{I}^{\dagger} \in \mathfrak{u s p}(28,28) .
$$

(3) $Y_{79}(2.20)$. It generates a $U(1)$ subgroup, corresponding to the compact Cartan of the $S L(2, \mathbb{R})$ factor group, appearing in $(2.14)$ and (2.15). The symmetry properties are:

$$
Y_{79}=Y_{79}^{T}, \quad Y_{79}^{\dagger}=-Y_{79} \Longrightarrow Y_{79} \in \mathfrak{u s p}(28,28) .
$$

(4) $Y_{I}$ with $I=80, \ldots, 106$, i.e., $Y_{\alpha+79}(2.21)$. The symmetry properties read as follows:

$$
\begin{gathered}
A_{\alpha}=A_{\alpha}^{T}, \quad A_{\alpha}^{\dagger}=A_{\alpha} \Longrightarrow Y_{\alpha+79}^{\dagger}=Y_{\alpha+79}, \quad Y_{\alpha+79}=-Y_{\alpha+79}^{T}, \\
Y_{\alpha+79} \in \mathfrak{u s p}(28,28) .
\end{gathered}
$$

(5) $Y_{I}$ with $I=107, \ldots, 133$, i.e., $Y_{\alpha+106}(2.22)$. The symmetry properties read as follows:

$Y_{\alpha+106}^{\dagger}=Y_{\alpha+106}, \quad Y_{\alpha+106}=-Y_{\alpha+106}^{T}, \quad Y_{\alpha+106} \in \mathfrak{u s p}(28,28)$. 
Thus, (2.38) results from (2.43) to (2.47).

As elucidated in the next section, the matrices $Y_{I}, I=80, \ldots, 133$ given by (2.21) and (2.22) are the Hermitian generators of the symmetric maximal non-compact (special Kähler) Riemannian coset (1.2), which is the classical vector multiplets' scalar manifold of the magical $\mathcal{N}=2, D=4$ MaxwellEinstein supergravity theory based on $\mathfrak{J}_{3}(\mathbb{O})[10]$. As given by $(2.27)$, the 27 matrices $A_{\alpha}$ are directly related to the invariant $d$-tensor of the $\mathbf{2 7}$ irrep. of $E_{6(-78)}$; they have been explicitly constructed in [12], to which the reader is addressed for further detail.

\section{Manifestly $\left[\left(E_{6(-78)} \times U(1)\right) / \mathbb{Z}_{3}\right]$-covariant coset construction}

The quotient manifold $\mathcal{M}(1.2)$ has rank 3 ; this means that the maximal dimension of the intersection between a Cartan subalgebra of $E_{7(-25)}$ and the generators of $\mathcal{M}$ itself is 3 . From the results reported above, the three generators of a Cartan subalgebra of $\mathcal{M}$ can be chosen to be the diagonal generators of the Jordan algebra $\mathfrak{J}_{3}(\mathbb{O})$ itself, namely $Y_{123}, Y_{132}$, and $Y_{133}$.

The coset $\mathcal{M}(1.2)$ is generated by the matrices $Y_{79+I},(2.21)$, and (2.22) with $I=1, \ldots, 54$. Through the exponential mapping, it can be defined as follows:

$$
\mathcal{M}:=\exp \left(\sum_{\alpha=1}^{27} x_{\alpha} Y_{106+\alpha}+y_{\alpha} Y_{79+\alpha}\right)
$$

with $x_{\alpha}, y_{\alpha}, \alpha=1, \ldots 27$, real parameters.

From the commutation relations of the matrices $Y$ 's, which can be easily computed by means of the Mathematica program provided in [12], it holds that:

$$
\left[Y_{51+\alpha}, Y_{106}\right]=-\sqrt{\frac{2}{3}} Y_{106+\alpha}, \quad\left[Y_{51+\alpha}, Y_{133}\right]=\sqrt{\frac{2}{3}} Y_{79+\alpha}
$$

The generators of $\mathfrak{e}_{6(-78)}$ which are not in $\mathfrak{f}_{4(-52)}$ mix the matrices $Y_{79+\alpha}$ with the $Y_{106+\alpha}$. Therefore, in order to make the complex structure of $\mathcal{M}$ 
manifest, it is necessary to introduce the following complex linear combinations of the matrices:

$$
\begin{aligned}
\zeta_{\alpha} & :=\frac{1}{\sqrt{2}}\left(Y_{79+\alpha}+\mathrm{i} Y_{106+\alpha}\right), \\
\bar{\zeta}_{\alpha} & :=\frac{1}{\sqrt{2}}\left(Y_{79+\alpha}-\mathrm{i} Y_{106+\alpha}\right) .
\end{aligned}
$$

This hints for the complex linear combinations of the parameters:

$$
\begin{aligned}
& z_{\alpha}:=\frac{1}{\sqrt{2}}\left(y_{\alpha}+\mathrm{i} x_{\alpha}\right), \\
& \bar{z}_{\alpha}:=\frac{1}{\sqrt{2}}\left(y_{\alpha}-\mathrm{i} x_{\alpha}\right),
\end{aligned}
$$

which allows one to rewrite (3.1) as:

$$
\mathcal{M}:=\exp \left(\sum_{\alpha=1}^{27} \bar{z}_{\alpha} \zeta_{\alpha}+z_{\alpha} \bar{\zeta}_{\alpha}\right)
$$

By introducing the 27-dimensional complex vector:

$$
z:=\sum_{\alpha=1}^{27} z_{\alpha} \vec{e}_{\alpha}
$$

and the $28 \times 28$ matrix:

$$
\mathcal{A}:=\left(\begin{array}{c|c}
-\sqrt{2} \sum_{\alpha=1}^{27} \bar{z}_{\alpha} A_{\alpha} & z \\
\hline z^{T} & 0
\end{array}\right)
$$

Equation (3.5) enjoys the simple form:

$$
\mathcal{M}:=\exp \left(\begin{array}{c|c}
0 & \mathcal{A} \\
\hline \mathcal{A}^{\dagger} & 0
\end{array}\right)=\left(\begin{array}{c|c}
\operatorname{Ch}\left(\sqrt{\mathcal{A} \mathcal{A}^{\dagger}}\right) & \mathcal{A} \frac{\operatorname{Sh}\left(\sqrt{\mathcal{A}^{\dagger} \mathcal{A}}\right)}{\sqrt{\mathcal{A}^{\dagger} \mathcal{A}}} \\
\hline \frac{\operatorname{Sh}\left(\sqrt{\mathcal{A} \mathcal{A}^{\dagger}}\right)}{\sqrt{\mathcal{A} \mathcal{A}^{\dagger}}} \mathcal{A}^{\dagger} & \operatorname{Ch}\left(\sqrt{\mathcal{A}^{\dagger} \mathcal{A}}\right)
\end{array}\right) .
$$


This is a Hermitian matrix, of the same form as the finite coset representative worked out [55] for the split (i.e., maximally non-compact) counterpart

$$
\mathcal{M}_{\mathcal{N}=8}=\frac{E_{7(7)}}{S U(8) / \mathbb{Z}_{2}},
$$

which is the scalar manifold of maximal $\mathcal{N}=8, D=4$ supergravity, associated to $\mathfrak{J}_{3}\left(\mathbb{O}_{S}\right)$. On the other hand, as a consequence of $(2.38), \mathcal{M}$ also is an element of $U S p(28,28)$, whereas $\mathcal{M}_{\mathcal{N}=8}$ is real.

By using the machinery of special Kähler geometry (see, e.g., [37,46,47]), the symplectic sections defining the symplectic frame associated to the coset parametrization introduced above can be directly read from (3.7)and (3.8):

$$
\mathcal{M}=:\left(\begin{array}{c|c}
u_{i}^{\Lambda}(z, \bar{z}) & v_{i \Lambda}(z, \bar{z}) \\
\hline v^{i \Lambda}(z, \bar{z}) & u_{\Lambda}^{i}(z, \bar{z})
\end{array}\right),
$$

where the symplectic index $\Lambda=0,1, \ldots 27$ (with 0 pertaining to the $\mathcal{N}=2$, $D=4$ graviphoton), and $i=\bar{\alpha}, 28$. Thus, the symplectic sections read (see, e.g., $[47,56]$; subscript " 28 " omitted):

$$
\begin{aligned}
f_{i}^{\Lambda} & :=\frac{1}{\sqrt{2}}(u+v)_{i}^{\Lambda}=\left(\bar{f}_{\bar{\alpha}}^{\Lambda}, f^{\Lambda}\right):=\left(\overline{\mathcal{D}}_{\bar{\alpha}} \bar{L}^{\Lambda}, L^{\Lambda}\right) \\
& =\exp \left(\frac{1}{2} K\right)\left(\overline{\mathcal{D}}_{\bar{\alpha}} \bar{X}^{\Lambda}, X^{\Lambda}\right) ; \\
h_{i \Lambda} & :=-\frac{\mathrm{i}}{\sqrt{2}}(u-v)_{i \Lambda}=\left(\bar{h}_{\bar{\alpha} \mid \Lambda}, h_{\Lambda}\right):=\left(\overline{\mathcal{D}}_{\bar{\alpha}} \bar{M}_{\Lambda}, M_{\Lambda}\right) \\
& =\exp \left(\frac{1}{2} K\right)\left(\overline{\mathcal{D}}_{\bar{\alpha}} \bar{F}_{\Lambda}, F_{\Lambda}\right),
\end{aligned}
$$

where $\mathcal{D}$ is the Kähler-covariant differential operator,

$$
\mathcal{V}:=\left(L^{\Lambda}, M_{\Lambda}\right)^{T}=\exp \left(\frac{1}{2} K\right)\left(X^{\Lambda}, F_{\Lambda}\right)^{T}
$$

is the symplectic vector of Kähler-covariantly holomorphic sections, and

$$
K:=-\ln \left[\mathrm{i}\left(\bar{X}^{\Lambda} F_{\Lambda}-X^{\Lambda} \bar{F}_{\Lambda}\right)\right]
$$

is the Kähler potential determining the corresponding geometry.

As announced, a key feature of (3.7) is that the matrix $\mathcal{A}$, generating the coset $\mathcal{M}(1.2)-(3.8)$, is written in terms of the invariant rank-3 $d$-tensor 
of the $\mathbf{2 7}$ fundamental irrep. of $E_{6(-78)}$, thus yielding a formalism with manifest $\left[\left(E_{6(-78)} \times U(1)\right) / \mathbb{Z}_{3}\right]$-covariance, which is the maximal compact possible symmetry of the framework under consideration. Within such a parametrization, the complex scalar fields of the corresponding $\mathcal{N}=2$ magic theory, coordinatizing $\mathcal{M}(1.2)$, are defined by (3.4), and summarized in vector notation by (3.6).

Furthermore, attention should be paid not to confuse this symplectic frame with the so-called " $4 D / 5 D$ special coordinates" symplectic frame (see, e.g., [49]), in which the holomorphic prepotential function $F$ exists and it is given by $\left(\left(X^{0}\right)^{2}\right.$ times) (2.29). Indeed, as commented below, $F$ simply does not exist in the symplectic frame under consideration (namely, $2 F=X^{\Lambda} F_{\Lambda}=0[57]$ ), and the $d$-tensor of the $\mathbf{2 7}$ of $E_{6(-26)}$ (appearing in (2.29)) is different from the $d$-tensor of $\mathbf{2 7}$ of $E_{6(-78)}$, appearing in the treatment of Section 2 and of the present section; such a difference is evident, e.g., when considering a manifestly $\left[G_{6} \times S O(1,1)\right]$-invariant formalism, as done, e.g., in $[38,44]$.

As mentioned in Section 2, by exploiting the expressions (2.25) and (2.26) of the non-compact generators of the relevant $\mathfrak{s l}(2, \mathbb{R})$ subalgebra, the maximal manifest $\left[\left(E_{6(-78)} \times U(1)\right) / \mathbb{Z}_{3}\right]$-covariance can be broken down to a manifest $\left[F_{4(-52)} \times U(1)\right]$-covariance (recall the maximal symmetric embedding (2.14) and (2.15)), in which (3.7) becomes:

$$
\mathcal{A}=\left(\begin{array}{c|c}
\frac{1}{\sqrt{3}} \bar{z}_{27} \tilde{I}-\sqrt{2} \sum_{a=1}^{26} \bar{z}_{a} A_{a} & z \\
\hline z^{T} & 0
\end{array}\right) .
$$

We note in passing that $F_{4(-52)}$ is particularly relevant, because it contains all the compact generators of $U S p(6,2)$, which is the maximal (noncompact) manifest covariance exhibited by the $d$-tensor of the $\mathbf{2 7}$ irrep. of $E_{6(-26)}$ constructed in [38]:

$$
\begin{aligned}
U S p(6,2) \cap U S p(8) \cap F_{4(-52)} & =U S p(6) \times U S p(2) \\
& \sim U S p(6) \times S U(2)=\operatorname{mcs}[U S p(6,2)] .
\end{aligned}
$$

\section{$3.1 \quad$ Remarks}

In order to gain more insight on the parametrization under consideration, it is useful to compare the infinitesimal element of the coset $\mathcal{M}(1.2)$, given 
by the $28 \times 28$ matrix (recall (3.8) and (3.7)):

$$
\ln \mathcal{M}:=\left(\begin{array}{c|c}
0 & \mathcal{A} \\
\hline \mathcal{A}^{\dagger} & 0
\end{array}\right),
$$

with an analogue expression, given by (6) of [48], which we recall here for ease of comparison:

$$
\mathcal{B}:=\left(\begin{array}{c|c|c|c}
0_{\beta}^{\alpha} & -t^{\prime \alpha} & d^{\alpha \beta \gamma} t_{\gamma} & 0^{\alpha} \\
\hline-t_{\beta} & 0 & 0_{\beta} & 0 \\
\hline d_{\alpha \beta \gamma} t^{\prime \gamma} & 0_{\alpha} & 0_{\alpha}^{\beta} & t_{\alpha} \\
\hline 0_{\beta} & 0 & t^{\prime \beta} & 0
\end{array}\right) .
$$

Following the treatment of [48], $\mathcal{B}$ is a real $28 \times 28$ matrix depending on $27+$ $27=54$ parameters, parametrizing the generators of the maximal symmetric non-compact pseudo-Riemannian rank-3 coset

$$
\widehat{\mathcal{M}}:=\frac{E_{7(7)}}{E_{6(6)} \times S O(1,1)},
$$

with signature $\left(-27,+{ }^{27}\right)$; in this case, the $d$-tensor appearing in (3.18) is the one pertaining to the $\mathbf{2 7}$ (or $\mathbf{2 7}$ ) irrep. of the split non-compact real form $E_{6(6)}$. On the other hand, by suitably replacing this latter by the $d$ tensor pertaining to the $\mathbf{2 7}$ (or $\mathbf{2 7}^{\prime}$ ) irrep. of the minimally non-compact real form $E_{6(-26)}$, the matrix $\mathcal{B}(3.18)$ can be regarded as parametrizing the generators of the maximal symmetric non-compact pseudo-Riemannian rank-3 coset

$$
\widetilde{\mathcal{M}}:=\frac{E_{7(-25)}}{E_{6(-26)} \times S O(1,1)},
$$

with signature $\left(-{ }^{43},+{ }^{11}\right)$; this pseudo-Riemannian counterpart of $(1.2)$ can also be regarded as the classical vector multiplets' scalar manifold of the magical octonionic $\mathcal{N}=2$ Maxwell-Einstein supergravity theory in $D=(4,0)$ dimensions, obtained from its $D=(4,1)$ uplift by timelike Kaluza-Klein reduction (see, e.g., [58]). Clearly, also other interpretations of $\mathcal{B}(3.18)$ are possible, within the maximal (symmetric) embeddings of (non-compact, real forms of) $E_{6} \times U(1)$ into (non-compact, real forms of) $E_{7}$ (see, e.g., [59]), but they are not relevant for the present investigation. Notice that in the above expressions (3.19) for $\widehat{\mathcal{M}}$ and (3.20) for $\widehat{\mathcal{M}}$ the issue of the presence of finite or discrete factors is not taken into account. 
We will now relate the matrix $\mathcal{B}(3.18)$ (which, within the interpretation (3.20), provides a manifestly $\left[E_{6(-26)} \times S O(1,1)\right]$-covariant parametrization of the generators of the coset $\widetilde{\mathcal{M}}$ ) to the matrix $\ln \mathcal{M}$ (3.17) (which provides a manifestly $\left[\left(E_{6(-78)} \times U(1)\right) / \mathbb{Z}_{3}\right]$-covariant parametrization of the generators of the $\operatorname{coset} \mathcal{M}(1.2))$.

(1) We start and move the vectors $t^{\prime}$ and $t$ from the diagonal blocks of $\mathcal{B}$ to the off-diagonal ones. In order to achieve this, a symplectic automorphism generated by the following matrix has to be performed:

$$
\mathcal{S}:=\left(\begin{array}{c|c|c|c}
I_{27} & 0 & 0 & 0 \\
\hline 0 & 0 & 0 & -1 \\
\hline 0 & 0 & I_{27} & 0 \\
\hline 0 & 1 & 0 & 0
\end{array}\right) .
$$

Thus, it follows that $\left(\mathcal{S}^{T} \Omega \mathcal{S}=\Omega\right)$

$$
\mathcal{S B S}^{-1}=\left(\begin{array}{c|c|c|c}
0_{\beta}^{\alpha} & 0^{\alpha} & d^{\alpha \beta \gamma} t_{\gamma} & -t^{\prime \alpha} \\
\hline 0_{\beta} & 0 & -t_{\beta}^{\prime} & 0 \\
\hline d_{\alpha \beta \gamma} t^{\prime \gamma} & -t_{\alpha} & 0_{\alpha}^{\beta} & 0_{\alpha} \\
\hline-t_{\beta} & 0 & 0_{\beta} & 0
\end{array}\right)
$$

(2) Then, it is necessary to make the following identification between the 27-dimensional vectors $t, t^{\prime}$ of $\mathcal{B}$ (3.18) and $z, \bar{z}$ defined by (3.4) and (3.6):

$$
\left\{\begin{array}{l}
t=-\bar{z} \\
t^{\prime}=-z
\end{array}\right.
$$

(3) By recalling the normalization of the $d$-tensor given by (2.27), it thus follows that $\mathcal{B}$ can be transformed into $\ln \mathcal{M}(3.17)$.

It is here worth remarking that an analytical direct exponentiation of the matrix $\mathcal{B}(3.8)$, which would yield an explicit symplectic frame, e.g., for the manifold (3.19) or (3.20), and which, through the 3-step procedure just mentioned, would provide a more explicit form of the treatment of Sections 2 and 3 , does not seem to be feasible (in [60] the exponentiation of $\mathcal{B}(3.8)$ with $t=0$ or, equivalently, $t^{\prime}=0$ has only been performed). It may be possible that a direct exponentiation of the matrix $\mathcal{B}(3.8)$ could be performed by exploiting the fundamental identity for the $d$-tensor of the symmetric coset. With the above normalization, such an identity can be derived from the 
treatment given in [61] (at least for $E_{6}$ ):

$$
d_{\alpha \beta \gamma} d^{\lambda \mu \gamma} d_{\mu \nu \rho}=d_{\nu \rho \alpha} \delta_{\beta}^{\lambda}+\frac{1}{3} d_{\nu \rho \beta} \delta_{\alpha}^{\lambda}-6 d_{\mu \nu \rho} T_{I \mid \alpha}^{\lambda} T_{\beta}^{I \mu},
$$

where as in the explicit treatment of Sections 2 and 3, Greek indices run over the fundamental $\mathbf{2 7}$ (or $\overline{\mathbf{2 7}}$ ) irrep., and capital Latin indices run over the adjoint 78 irrep. of $E_{6}$; the $T_{I \mid \alpha \beta}$ 's denote the realization of the generators of $E_{6}$ in its 27 irrep. (see, e.g., [61]), and they are, e.g., proportional to the $\phi_{I}$ 's (2.23) appearing in the matrices $Y_{I}(2.19)$. We note that the complete symmetrization of covariant indices of the identity (3.24) yields the well known identity:

$$
d_{(\alpha \beta \mid \gamma} d^{\lambda \mu \gamma} d_{\mu \mid \nu \rho)}=\frac{4}{3} d_{(\nu \rho \beta} \delta_{\alpha)}^{\lambda} .
$$

We leave for the future the interesting task of exploiting the identity (3.24) and/or spectral techniques in order to perform the exponentiation of the matrix $\mathcal{B}(3.8)$, and thus to determine a more explicit expression of the maximally manifestly covariant symplectic frame introduced in Sections 2 and 3 .

\section{The Iwasawa decomposition}

In this section, we are going to construct, along the lines of [22], another parametrization for the coset $\mathcal{M}(1.2)$, by exploiting the Iwasawa decomposition, which in this case turns out to be manifestly $S O(8)$-covariant, thus providing a manifestly triality symmetric description of the rank-3 coset $\mathcal{M}$. Within this treatment, we will denote by $\mathfrak{P}$ the Lie algebra of the coset $\mathcal{M}$, namely the complement in $\mathfrak{e}_{7(-25)}$ to its maximal compact subalgebra $\mathfrak{T}:=\mathfrak{e}_{6(-78)} \oplus \mathfrak{u}(1)$.

As the first step, one needs to determine a maximal non-compact Cartan subalgebra $\mathfrak{H}_{3}$. As observed at the start of Section 3, a possible choice is:

$$
\mathfrak{H}_{3}:=\left\langle Y_{123}, Y_{132}, Y_{133}\right\rangle_{\mathbb{R}} \subset \mathfrak{P},
$$

generated by the diagonal elements of $\mathfrak{J}_{3}(\mathbb{O})$.

Next, a basis of $54-3=51$ positive roots of $\mathfrak{P}$ with respect to $\mathfrak{H}_{3}$ (4.1) is to be determined.

If the adjoint action of $\mathfrak{H}_{3}$ on $\mathfrak{e}_{7(-25)}$ is simultaneously diagonalized, we expect to be able to find only 102 non-vanishing vectors in $\mathbb{R}^{3}$. This follows 
from the fact that, apart from $\mathfrak{H}_{3}$ itself, $\mathfrak{H}_{3}$ commutes with a 28 -dimensional subalgebra $\mathfrak{S} \simeq \mathfrak{s o}(8)$ of $\mathfrak{T}$; this can be easily understood by the following argument. By denoting with $\oplus_{s}$ the semi-direct sum of algebras, due to the symmetric nature of the embedding determining the coset $\mathcal{M}(1.2)$, the structure of the Cartan decomposition of $\mathfrak{e}_{7(-25)}=\mathfrak{T} \oplus_{s} \mathfrak{P}$ reads:

$$
[\mathfrak{T}, \mathfrak{T}] \subseteq \mathfrak{T}, \quad[\mathfrak{P}, \mathfrak{P}] \subseteq \mathfrak{T}, \quad[\mathfrak{T}, \mathfrak{P}] \subseteq \mathfrak{P} .
$$

As usual, the last relation implies that $\mathfrak{P}$ is a representation space for $\mathfrak{T}$, which in general will decompose in irreducible subspaces. In particular, $\mathfrak{P}$ is a representation space for the $\mathfrak{f}_{4(-52)}$ subalgebra of $\mathfrak{T}$. As it is well known, $\mathfrak{f}_{4(-52)}$ is the Lie algebra of the group $\operatorname{Aut}\left(\mathfrak{J}_{3}(\mathbb{O})\right)$; in turn the subalgebra of $\mathfrak{f}_{4(-52)}$ which keeps the diagonal elements of $\mathfrak{J}_{3}(\mathbb{O})$ fixed is precisely $\mathfrak{s o}(8)$, namely the Lie algebra of the automorphism group $\operatorname{Aut}(\mathbf{t}(\mathbb{O}))$ of the normed triality on $\mathbb{O}$ (see, e.g., [62]). Therefore, since $\mathfrak{H}_{3}$ has been selected exactly as the subalgebra corresponding to the diagonal elements of $\mathfrak{J}_{3}(\mathbb{O})$, it has to commute with an $\mathfrak{s o}(8)$ subalgebra of $\mathfrak{f}_{4(-52)}$; this can indeed be checked by inspecting the structure of the roots. Following [34,35], a Cartan subalgebra $\mathfrak{H}_{7} \subset \mathfrak{e}_{7(-25)}$ can be obtained by adding the space $\mathfrak{H}_{4}$ generated by the four matrices $Y_{i}, i=1,6,15,36$ (recall (2.19)) to $\mathfrak{H}_{3}$. Then, the computation of the roots with respect to this system exactly yields 28 roots with vanishing components in the subspace $\mathfrak{H}_{3}$; these generate the $\mathfrak{H}_{3}$-preserving Lie algebra [34]:

$$
\mathfrak{S}:=\left\langle Y_{1}, \ldots Y_{21}, Y_{30}, \ldots, Y_{36}\right\rangle_{\mathbb{R}}=\mathfrak{s o}(8),
$$

whose $\left\langle Y_{i}\right\rangle_{i=1,6,15,36}$ is thus a Cartan subalgebra. Note that in $[34,35]$ a completion of $\left\langle Y_{i}\right\rangle_{i=1,6,15,36}$ to $\mathfrak{s o}(8) \neq \mathfrak{S}$ (4.3) was worked out, but this is irrelevant for the present investigation.

As a consequence, it holds:

$$
\left.a d_{\mathfrak{H}_{3}}\right|_{\mathfrak{S} \oplus \mathfrak{H}_{3}}=0,
$$

so that 31 eigenvalues vanish in $\mathbb{R}^{3}$, and thus only at most $133-31=102$ can be non-vanishing, as expected.

Let us show that actually all the remaining 102 eigenvalues of $a d_{\mathfrak{H}_{3}}$ on $\mathfrak{e}_{7(-25)}$ are non-vanishing. First, we can write:

$$
\begin{aligned}
\mathfrak{T} & =\mathfrak{S} \oplus_{s} \mathfrak{T}^{\prime}, \\
\mathfrak{P} & =\mathfrak{H}_{3} \oplus_{s} \mathfrak{P}^{\prime},
\end{aligned}
$$


with $\operatorname{dim}_{\mathbb{R}} \mathfrak{T}^{\prime}=\operatorname{dim}_{\mathbb{R}} \mathfrak{P}^{\prime}=51$. Now,

$$
a d_{\mathfrak{H}_{3}}: \mathfrak{T}^{\prime} \oplus_{s} \mathfrak{P}^{\prime} \longrightarrow \mathfrak{T}^{\prime} \oplus_{s} \mathfrak{P}^{\prime}
$$

Indeed, $[\mathfrak{P}, \mathfrak{P}] \subseteq \mathfrak{T}(4.2)$ implies $\left[\mathfrak{H}_{3}, \mathfrak{P}^{\prime}\right] \subseteq \mathfrak{T}$. Let $\langle,\rangle_{c k}$ be the CartanKilling product; then, its restriction to $\mathfrak{T}$ has a definite signature, usually chosen to be negative. The fact that $\left[\mathfrak{S}, \mathfrak{H}_{3}\right]=0$ implies:

$$
\left\langle\mathfrak{S},\left[\mathfrak{H}_{3}, \mathfrak{P}^{\prime}\right]\right\rangle_{c k}=-\left\langle\left[\mathfrak{H}_{3}, \mathfrak{S}\right], \mathfrak{P}^{\prime}\right\rangle_{c k}=0 \Rightarrow\left[\mathfrak{H}_{3}, \mathfrak{P}^{\prime}\right] \in \mathfrak{T}^{\prime}
$$

Next, from $[\mathfrak{T}, \mathfrak{P}] \subseteq \mathfrak{P}(4.2)$ it follows that $\left[\mathfrak{H}_{3}, \mathfrak{T}^{\prime}\right] \subseteq \mathfrak{P}$. As the CartanKilling form is strictly positive on $\mathfrak{P}$, and $\mathfrak{H}_{3}$ is Abelian, one obtains:

$$
\left\langle\mathfrak{H}_{3},\left[\mathfrak{H}_{3}, \mathfrak{T}^{\prime}\right]\right\rangle_{c k}=-\left\langle\left[\mathfrak{H}_{3}, \mathfrak{H}_{3}\right], \mathfrak{T}^{\prime}\right\rangle_{c k}=0 \Rightarrow\left[\mathfrak{H}_{3}, \mathfrak{T}^{\prime}\right] \subseteq \mathfrak{P}^{\prime}
$$

In this way, one can conclude that the set $\mathcal{W}$ of the remaining 102 roots of $\mathfrak{e}_{7(-25)}$ has eigenspaces in $\mathfrak{T}^{\prime} \oplus_{s} \mathfrak{P}^{\prime}$. Thus, each eigenvector has the form $\lambda_{A}:=t_{A}+p_{A}, A=1, \ldots, 102$, where $t_{A} \in \mathfrak{T}^{\prime}$ and $p_{A} \in \mathfrak{P}^{\prime}$ are both nonvanishing and uniquely determined by $\lambda_{A}$. Let us suppose that one of the roots $r_{A_{0}} \in \mathcal{W}$ vanishes: $r_{A_{0}}=0$. This would imply that $a d_{\mathfrak{H}_{3}}\left(p_{A_{0}}\right)=0$. But, in turn, this would also mean $p_{A_{0}} \in \mathfrak{H}_{3}$ (as $\mathfrak{H}_{3}$ is a maximal Cartan subalgebra in $\mathfrak{P}$ ), which cannot be the case, since $\mathfrak{H}_{3} \cap \mathfrak{P}^{\prime}=0$.

Keeping in mind that we are considering the roots of $\mathfrak{e}_{7(-25)}$ relative to the choice (4.1) of $\mathfrak{H}_{3}$, we can thus conclude that all 102 roots in $\mathcal{W}$ are non-vanishing. Let us now fix a choice of 51 positive roots $\mathcal{W}_{+}$so that $\mathcal{W}=\mathcal{W}_{+} \cup \mathcal{W}_{-}$. The corresponding eigenspaces are one-dimensional, and they are generated by the eigenvectors $\lambda_{i}^{+}, i=1, \ldots, 51$, with eigenvalues $r_{i} \in \mathcal{W}_{+}$. We can write in a unique way:

$$
\lambda_{i}^{+}=p_{i}+t_{i}
$$

implying that:

$$
\lambda_{i}^{-}:=p_{i}-t_{i}
$$

are eigenvalues of $-r_{i} \in \mathcal{W}_{-}$. 
Finally, by renaming $h_{1}:=Y_{123}, h_{2}:=Y_{132}$ and $h_{3}:=Y_{133}$, the Iwasawa decomposition of the coset $\mathcal{M}(1.2)$ can be written as:

$$
\mathcal{M}:=\exp \left(x_{1} h_{1}+x_{2} h_{2}+x_{3} h_{3}\right) \exp \left(\sum_{i=1}^{51} y_{i} \lambda_{i}^{+}\right) .
$$

which exhibits a manifest $S O(8)$-covariance. We anticipate that $\mathfrak{s o}(8)$ is the Lie algebra of $\operatorname{Aut}(\mathbf{t}(\mathbb{O}))=\operatorname{Spin}(8)$, namely the automorphism group of the normed triality $\mathbf{t}(\mathbb{O})$ over the division algebra of octonions $\mathbb{O}$ (see, e.g., [62]):

$$
\mathfrak{s o}(8)=\mathfrak{A u t}(\mathbf{t}(\mathbb{O}))=: \mathfrak{t r i}(\mathbb{O}) ;
$$

see the discussion in Section 5 .

\subsection{SO (8)-Triality}

Now, we want study the $S O(8)$-covariance of the Iwasawa parametrization (4.12) in more detail.

First, as pointed out above, the elements $h_{1}, h_{2}, h_{3}$ of the Cartan subalgebra $\mathfrak{H}_{3}$ commute with $S O(8)$, and it follows that they are three $S O(8)$ singlets. Thus, the 51 -dimensional linear space $\Lambda_{+}$generated by the positive roots $\mathcal{W}_{+}$is invariant under the (adjoint) action of $S O(8)$, and it decomposes into irreps. of $S O(8)$ as:

$$
\Lambda_{+}=\mathbf{1}^{3}+\mathbf{8}_{v}^{2}+\mathbf{8}_{c}^{2}+\mathbf{8}_{s}^{2} .
$$

The manifestly triality-symmetric decomposition (4.14) can be proven by means of the following general argument. Let us fix an orthonormal basis $L_{1}, \ldots, L_{7}$ of $\mathbb{R}^{7}$. Then, the $(133-7) / 2=63$ positive roots of $E_{7(-25)}$ can be represented as (see, e.g., [63], p. 333):

$$
\begin{aligned}
& L_{m} \pm L_{n}, \quad 1 \leq n<m \leq 6 ; \quad \sqrt{2} L_{7} ; \\
& \frac{ \pm L_{1} \pm L_{2} \pm L_{3} \pm L_{4} \pm L_{5} \pm L_{6}+\sqrt{2} L_{7}}{2}, \quad \text { odd number of }- \text { signs. }
\end{aligned}
$$

Among these, the $(28-4) / 2=12$ roots:

$$
\mu_{m n}^{ \pm}:=L_{m} \pm L_{n}, \quad 1 \leq n<m \leq 4
$$


are the positive roots of $\mathfrak{s o}(8) . \mu_{m n}^{ \pm}$(4.16) provide a representation of the algebra $\mathfrak{s o}(8)$ over the linear space generated by the remaining 51 roots in the usual way: if, consistent with (4.10), we call $\lambda_{i}^{+}$the 51 complementary roots, then the linear operators $\mu_{m n}^{ \pm}$and their corresponding adjoint $\tilde{\mu}_{m n}^{ \pm}$ are defined by:

$$
\begin{aligned}
& \mu_{m n}^{ \pm}\left(\lambda_{i}^{+}\right):=\lambda_{i}^{+}+\left(L_{m} \pm L_{n}\right), \\
& \tilde{\mu}_{m n}^{ \pm}\left(\lambda_{i}^{+}\right):=\lambda_{i}^{+}-\left(L_{m} \pm L_{n}\right),
\end{aligned}
$$

where the result is intended to be zero when the vectors on the right-hand side are not roots.

This procedure allows to identify exactly 9 invariant subspaces of $\Lambda_{+}$:

(1) The three spaces respectively generated by $L_{6}+L_{5}, L_{6}-L_{5}$ and $\sqrt{2} L_{7}$ are one-dimensional invariant subspaces defining a $\mathbf{1}^{3}$ representation (sum of $3 S O(8)$-singlets).

(2) The two 8-dimensional spaces $V_{5}$ and $V_{6}$, respectively generated by the basis:

$$
\begin{aligned}
& \left\{L_{5} \pm L_{n}\right\}_{n=1}^{4} ; \\
& \left\{L_{6} \pm L_{n}\right\}_{n=1}^{4}
\end{aligned}
$$

are both representations with weights $\pm L_{n}$, and thus correspond to two copies of the vector representation $\mathbf{8}_{v}$.

(3) The two 8-dimensional spaces $C_{+}$and $C_{-}$, respectively generated by the basis:

$$
\begin{aligned}
& \frac{ \pm L_{1} \pm L_{2} \pm L_{3} \pm L_{4}+L_{5}-L_{6}+\sqrt{2} L_{7}}{2} \\
& \frac{ \pm L_{1} \pm L_{2} \pm L_{3} \pm L_{4}-L_{5}+L_{6}+\sqrt{2} L_{7}}{2}
\end{aligned}
$$

(with an even number of - signs) are both representations with weights $\frac{ \pm L_{1} \pm L_{2} \pm L_{3} \pm L_{4}}{2}$ (with an even number of - signs), thus providing two copies of the chiral spinor representation $\mathbf{8}_{c}$.

(4) The two 8-dimensional spaces $S_{+}$and $S_{-}$, respectively generated by the basis:

$$
\begin{aligned}
& \frac{ \pm L_{1} \pm L_{2} \pm L_{3} \pm L_{4}+L_{5}+L_{6}+\sqrt{2} L_{7}}{2} \\
& \frac{ \pm L_{1} \pm L_{2} \pm L_{3} \pm L_{4}-L_{5}-L_{6}+\sqrt{2} L_{7}}{2}
\end{aligned}
$$


(with an odd number of - signs) are both representations with weights $\frac{ \pm L_{1} \pm L_{2} \pm L_{3} \pm L_{4}}{2}$ (with an odd number of - signs), thus providing two copies of the chiral spinor representation $\mathbf{8}_{s}$ (conjugate of $\boldsymbol{8}_{c}$ ).

This implies (4.14), in which the $S O(8)$-triality is manifest. It is worth remarking that the appearance of the square for the three 8 irreps. in (4.14) is a consequence of the complex (in particular, special Kähler, as mentioned in previous sections) structure of the $\operatorname{coset} \mathcal{M}(1.2)$.

\subsection{Group theory}

In the Iwasawa parametrization of $\mathcal{M}(1.2)$ worked out in Section 4, the resulting maximal manifest covariance group is nothing but the $S O(8)$ group (uniquely determined in $E_{7(-25)}$; see Sections 4.2 .1 and 4.2.2) preserving the diagonal elements in the rank-3 simple Jordan algebra $\mathfrak{J}_{3}(\mathbb{O})$. As clearly evident form the chain (4.26) of embeddings, such an $S O(8)$ is placed as follows:

$$
S O(8) \subset\left[(S O(10) \times U(1)) \cap F_{4(-52)}\right] .
$$

As given by $(4.13)$, it shares the same algebra $\mathfrak{s o}(8)=\mathfrak{t r i}(\mathbb{O})$ with the automorphism group Aut $(\mathbf{t}(\mathbb{O}))=\operatorname{Spin}(8)$ of the normed triality over the octonions $(\mathbb{O}$ [62]. Furthermore, it is worth remarking that such an $S O(8)$ recently appeared as the stabilizer of the BPS generic charge orbit in the two-centered extremal black hole solutions of $\mathcal{N}=2, D=4$ exceptional supergravity; see Table 7 [17].

We also note that, at the level of (manifest) covariance, the Iwasawa parametrization of $\mathcal{M}(1.2)$ worked out in Section 4 differs from the Iwasawa parametrization of $\mathcal{M}_{\mathcal{N}=8}$ (3.9) studied in [22], whose manifest maximal (non-compact) covariance is $S L(7, \mathbb{R})$, with maximal compact subgroup $S O(7)$.

\subsubsection{A first chain of embeddings}

The chain of maximal symmetric embeddings relevant for the study of the maximal manifest covariance of the Iwasawa parametrization (4.12) of the irreducible Riemannian globally symmetric rank-3 symmetric special Kähler coset $\mathcal{M}$ (1.2) reads as follows (see, e.g., [59]): 
(1) in the compact case:

$$
\begin{aligned}
E_{7(-25)} & \supset E_{6(-78)} \times U(1)^{\prime} \\
& \supset S O(10) \times U(1)^{\prime} \times U(1)^{\prime \prime} \\
& \supset S O(8) \times U(1)^{\prime} \times U(1)^{\prime \prime} \times U(1)^{\prime \prime \prime}
\end{aligned}
$$

(2) in the relevant (namely, minimally) non-compact case:

$$
\begin{aligned}
E_{7(-25)} & \supset E_{6(-26)} \times S O(1,1)^{\prime} \\
& \supset S O(9,1) \times S O(1,1)^{\prime} \times S O(1,1)^{\prime \prime} \\
& \supset S O(8) \times S O(1,1)^{\prime} \times S O(1,1)^{\prime \prime} \times S O(1,1)^{\prime \prime \prime} .
\end{aligned}
$$

In the last line of $(4.27)$ the first two $S O(1,1)$ factors have the physical meaning of "extra" $T$-dualities generated by the Kaluza-Klein reductions, respectively $D=5 \rightarrow D=4$, and $D=6 \rightarrow D=5$.

Correspondingly, the adjoint irrep. 133 of $E_{7(-25)}$ branches as (subscripts denote $U(1)$-charges or $S O(1,1)$-weights, for (4.26) and (4.27), respectively, throughout; see, e.g., [32]):

$$
\begin{aligned}
\mathbf{1 3 3}= & \mathbf{7 8}_{0}+\mathbf{1}_{0}+\mathbf{2 7}_{-2}+\mathbf{2 7}_{+2}^{\prime} \\
= & \mathbf{1}_{0,0}+\mathbf{1 6}_{0,-3}+\mathbf{1 6}_{0,+3}^{\prime}+\mathbf{4 5 _ { 0 , 0 }}+\mathbf{1}_{0,0} \\
& +\mathbf{1}_{-2,+4}+\mathbf{1 0}_{-2,-2}+\mathbf{1} \mathbf{6}_{-2,+1} \\
& +\mathbf{1}_{+2,-4}+\mathbf{1 0}_{+2,+2}+\mathbf{1 6}_{+2,-1}^{\prime} \\
= & \mathbf{1}_{0,0,0}+\mathbf{8}_{c, 0,-3,1}+\mathbf{8}_{s, 0,-3,-1}+\mathbf{8}_{c, 0,+3,-1}+\mathbf{8}_{s, 0,+3,+1} \\
& +\mathbf{1}_{0,0,0}+\mathbf{8}_{v, 0,0,+2}+\mathbf{8}_{v, 0,0,-2}+\mathbf{2} \mathbf{8}_{0,0,0}+\mathbf{1}_{0,0,0} \\
& +\mathbf{1}_{-2,+4,0}+\mathbf{1}_{-2,-2,+2}+\mathbf{1}_{-2,-2,-2}+\mathbf{8}_{v,-2,-2,0} \\
& +\mathbf{8}_{c,-2,+1,+1}+\mathbf{8}_{s,-2,+1,-1} \\
& +\mathbf{1}_{+2,-4,0}+\mathbf{1}_{+2,+2,-2}+\mathbf{1}_{+2,+2,+2}+\mathbf{8}_{v,+2,+2,0} \\
& +\mathbf{8}_{c,+2,-1,-1}+\mathbf{8}_{s,+2,-1,+1}
\end{aligned}
$$

Recalling the treatment Section 4, in line (4.28) one can recognize:

$$
\begin{aligned}
\mathfrak{T} & :=\mathbf{A d j}\left(E_{6} \times U(1)\right)=\mathbf{7 8}_{0}+\mathbf{1}_{0} ; \\
\mathfrak{P} & :=\mathbf{2 7}_{-2}+\mathbf{2} \mathbf{7}_{+2}^{\prime},
\end{aligned}
$$

where, by the definitions introduced in Section $4, \mathfrak{P}$ denotes (the irreducible decomposition of) the Lie algebra of the coset $\mathcal{M}(1.2)$ (as representation 
space of $\mathfrak{T})$. Furthermore, $\mathbf{2 7}_{-2}+\mathbf{2 7}_{+2}^{\prime}$ manifestly shows the complex (special Kähler) structure of $\mathcal{M}$ itself, which is then spoiled by the further subsequent branchings needed for the Iwasawa parametrization (4.12).

Furthermore, the lines (4.29) and (4.30) give the $S O(8) \times[U(1)]^{3}$ (or $\left.S O(8) \times[S O(1,1)]^{3}\right)$ irreducible branching of the 79 compact generators of $E_{7(-25)}$, namely of the generators of its maximal compact subgroup $E_{6(-78)} \times$ $U(1)$. On the other hand, the lines (4.31) and (4.32) give the $S O(8) \times$ $[U(1)]^{3} \quad\left(\right.$ or $\left.S O(8) \times[S O(1,1)]^{3}\right) \quad$ irreducible branching of the 54 non-compact generators of $E_{7(-25)}$, namely of the generators of $\mathcal{M}$ itself. In particular, recalling the definitions of Section 4:

$$
\begin{aligned}
\mathfrak{T}^{\prime}:= & \mathbf{1}_{0,0,0}+\mathbf{8}_{c, 0,-3,1}+\mathbf{8}_{s, 0,-3,-1}+\mathbf{8}_{c, 0,+3,-1}+\mathbf{8}_{s, 0,+3,+1} \\
& +\mathbf{1}_{0,0,0}+\mathbf{8}_{v, 0,0,+2}+\mathbf{8}_{v, 0,0,-2}+\mathbf{1}_{0,0,0} \\
\mathfrak{S}:= & \mathbf{A d j}(S O(8))=\mathbf{2 8}_{0,0,0} .
\end{aligned}
$$

$\mathfrak{T}^{\prime}$ is the Lie algebra of the non-maximal (and non-symmetric) $\operatorname{coset}\left(\operatorname{dim}_{\mathbb{R}}=\right.$ $51)$ :

$$
\frac{E_{6(-78)} \times U(1)^{\prime}}{S O(8)}=\frac{E_{6(-78)}}{S O(8)} \times U(1)^{\prime}
$$

or, in the choice of chain (4.27), of its relevant (i.e., minimally) non-compact form:

$$
\frac{E_{6(-26)} \times S O(1,1)^{\prime}}{S O(8)}=\frac{E_{6(-26)}}{S O(8)} \times S O(1,1)^{\prime} .
$$

Out of the six $S O(8)$-singlets:

$$
\mathbf{1}_{-2,+4,0}, \mathbf{1}_{-2,-2,+2}, \mathbf{1}_{-2,-2,-2}, \mathbf{1}_{+2,-4,0}, \mathbf{1}_{+2,+2,-2}, \mathbf{1}_{+2,+2,+2}
$$

in lines (4.31) and (4.32), three linear combinations generate $\mathfrak{H}_{3}$, whereas the remaining linear combinations, orthogonal with respect to the CartanKilling form, together with the manifestly $S O(8)$-triality-symmetric branching:

$$
\begin{aligned}
& \mathbf{8}_{v,-2,-2,0}+\mathbf{8}_{c,-2,+1,+1}+\mathbf{8}_{s,-2,+1,-1} \\
& \quad+\mathbf{8}_{v,+2,+2,0}+\mathbf{8}_{c,+2,-1,-1}+\mathbf{8}_{s,+2,-1,+1}
\end{aligned}
$$

of lines (4.31) and (4.32), generate $\mathfrak{P}^{\prime}$. 
Analogously, the smallest non-trivial symplectic irrep., namely the fundamental $\mathbf{5 6}$ of $E_{7(-25)}$, branches as (see, e.g., [32]):

$$
\begin{aligned}
\mathbf{5 6}= & \mathbf{2 7}_{+1}+\mathbf{2 7}_{-1}^{\prime}+\mathbf{1}_{+3}+\mathbf{1}_{-3} \\
= & \mathbf{1}_{+1,+4}+\mathbf{1 0}_{+1,-2}+\mathbf{1 6}_{+1,+1} \\
& +\mathbf{1}_{-1,-4}+\mathbf{1 0}_{-1,+2}+\mathbf{1 6}_{-1,-1}^{\prime} \\
& +\mathbf{1}_{+3,0}+\mathbf{1}_{-3,0} \\
= & \mathbf{1}_{+1,+4,0}+\mathbf{1}_{+1,-2,+2}+\mathbf{1}_{+1,-2,-2}+\mathbf{8}_{v,+1,-2,0}+\mathbf{8}_{c,+1,+1,+1} \\
& +\mathbf{8}_{s,+1,+1,-1}+\mathbf{1}_{-1,-4,0}+\mathbf{1}_{-1,+2,-2}+\mathbf{1}_{-1,+2,+2}+\mathbf{8}_{v,-1,+2,0} \\
& +\mathbf{8}_{c,-1,-1,-1}+\mathbf{8}_{s,-1,-1,+1}+\mathbf{1}_{+3,0,0}+\mathbf{1}_{-3,0,0}
\end{aligned}
$$

It is instructive to analyze the branchings (4.41)-(4.43) more in depth.

From the structure of the matrices $Y_{I}(2.19)$ and $(2.20), I=1, \ldots, 79$, the structure of the first branching (4.41) is evident, where the subscripts denote the charge (weight) with respect to $U(1)^{\prime}\left(S O(1,1)^{\prime}\right)$. Next, let us look at the $\mathbf{2 7}$ irrep. of $E_{6}$; which is realized over the 27-dimensional linear space of octonionic Hermitian matrices underlying the exceptional Jordan algebra $\mathfrak{J}_{3}(\mathbb{O})$. It decomposes as follows:

$$
\left(\begin{array}{ccc}
a & X & Y \\
X^{*} & b & Z \\
Y^{*} & Z^{*} & c
\end{array}\right)=\left(\begin{array}{ccc}
0 & X & Y \\
X^{*} & 0 & 0 \\
Y^{*} & 0 & 0
\end{array}\right)+\left(\begin{array}{ccc}
0 & 0 & 0 \\
0 & b & Z \\
0 & Z^{*} & c
\end{array}\right)+\left(\begin{array}{ccc}
a & 0 & 0 \\
0 & 0 & 0 \\
0 & 0 & 0
\end{array}\right)
$$

where $a, b, c$ are the real numbers and $X, Y, Z$ are the real (in the linear sense) octonions. As hinted in (4.44), this yields a decomposition $\mathbf{2 7}=\mathbf{1 6}+\mathbf{1 0}+\mathbf{1}$ of invariant spaces under the maximal symmetric subgroup $R:=S O(10) \times$ $U(1)^{\prime \prime}$ of $E_{6}$ (we consider, without loss of any generality, the compact chain (4.26) of embeddings). Indeed, the one-dimensional space $\mathbf{1}$ is easily seen to be invariant under $R$. As the spaces in the decomposition (4.44) are orthogonal with respect to the trace product (which is preserved by $R$ ), its complement is also $R$-invariant. On the other hand, the 16-dimensional subspace defines the largest subalgebra in $\mathfrak{J}_{3}(\mathbb{O})$ complementary to the onedimensional space $\mathbf{1}$. This proves our assertion.

The $U(1)^{\prime \prime}$-charges of the spaces in the right-hand side of (4.44) can be determined by noting that $U(1)^{\prime \prime} \nsubseteq F_{4(-52)}$. From the treatment of Section 2 , the Lie algebra $\mathfrak{e}_{6(-78)}$ is obtained by adding the left (or right) action of $\mathfrak{J}_{3}^{\prime}(\mathbb{O})$ on $\mathfrak{J}_{3}(\mathbb{O})$ (where the prime here denotes the matrix tracelessness). This means that the generator of $U(1)^{\prime \prime}$ must be realized by a traceless 
matrix $C_{U(1)^{\prime \prime}}$ in $\mathfrak{J}_{3}(\mathbb{O})$ that by left Jordan-multiplication acts proportionally to the identity on the three subspaces of the decomposition (4.44). This implies that:

$$
C_{U(1)^{\prime \prime}}=\left(\begin{array}{ccc}
2 \gamma & 0 & 0 \\
0 & -\gamma & 0 \\
0 & 0 & -\gamma
\end{array}\right)
$$

Writing (4.44) as $V_{27}=V_{16}+V_{10}+V_{1}$, we see that:

$$
C_{U(1)^{\prime \prime}} \circ V_{16}=\frac{\gamma}{2} V_{16}, \quad C_{U(1)^{\prime \prime}} \circ V_{10}=-\gamma V_{10}, \quad C_{U(1)^{\prime \prime}} \circ V_{1}=2 \gamma V_{1} .
$$

By choosing the normalization of the charges in such the way that $\exp \left(x C_{U(1)^{\prime \prime}}\right)$ has period $2 \pi$, one then obtains:

$$
27=16_{1}+10_{-2}+1_{4}
$$

which matches the convention, e.g., of [32]. Obviously, $U(1)^{\prime \prime}$ commutes with $U(1)^{\prime}$; therefore (4.42) is obtained.

For the last branching (4.43), the decompositions of $\mathbf{1 0}$ and $\mathbf{1 6}$ have to be analyzed. As $S O(8)$ leaves the diagonal matrices of $\mathfrak{J}_{3}(\mathbb{O})$ invariant, it follows that under its action the space $V_{10}$ decomposes as $V_{10}=V_{8}+V_{1, I}+$ $V_{1, I I}$ in the following way:

$$
\left(\begin{array}{ccc}
0 & 0 & 0 \\
0 & b & Z \\
0 & Z^{*} & c
\end{array}\right)=\left(\begin{array}{ccc}
0 & 0 & 0 \\
0 & 0 & Z \\
0 & Z^{*} & 0
\end{array}\right)+\left(\begin{array}{ccc}
0 & 0 & 0 \\
0 & b & 0 \\
0 & 0 & 0
\end{array}\right)+\left(\begin{array}{ccc}
0 & 0 & 0 \\
0 & 0 & 0 \\
0 & 0 & c
\end{array}\right)
$$

In order to determine the $U(1)^{\prime \prime \prime}$ charges, we again observe that $U(1)^{\prime \prime \prime} \varsubsetneqq$ $F_{4(-52)}$. Moreover, the $U(1)^{\prime \prime \prime}$ charge of $\mathbf{1}_{1,4}$ in (4.42) must be zero and, therefore, the $U(1)^{\prime \prime \prime}$ generator must be realized by a matrix of the form:

$$
C_{U(1)^{\prime \prime \prime}}=\left(\begin{array}{ccc}
0 & 0 & 0 \\
0 & \gamma^{\prime} & 0 \\
0 & 0 & -\gamma^{\prime}
\end{array}\right)
$$

By choosing the normalization as before in such a way that the period of $\exp \left(x C_{U(1)^{\prime \prime \prime}}\right)$ is $2 \pi$, one can fix $\gamma^{\prime}=2$, and the charges turn out to be 0 , 2 and -2 for $V_{8}, V_{1, I}$ and $V_{1, I I}$, respectively. Since $V_{8}$ is contained in the 
vector representation $V_{10}$ of $S O(10)$, it has to correspond to the vector rep. $\mathbf{8}_{v}$ of $S O(8)$, so that:

$$
\mathbf{1 0}=\mathbf{8}_{v, 0}+\mathbf{1}_{2}+\mathbf{1}_{-2} .
$$

The charge operator $C_{U(1)^{\prime \prime \prime}}(4.49)$ splits $V_{16}$ into eigenspaces $V_{8}^{+}$and $V_{8}^{-}$ with eigenvalues 1 and -1 , respectively:

$$
\left(\begin{array}{ccc}
0 & X & Y \\
X^{*} & 0 & 0 \\
Y^{*} & 0 & 0
\end{array}\right)=\left(\begin{array}{ccc}
0 & X & 0 \\
X^{*} & 0 & 0 \\
0 & 0 & 0
\end{array}\right)+\left(\begin{array}{ccc}
0 & 0 & Y \\
0 & 0 & 0 \\
\boldsymbol{8}_{c} & & 0
\end{array}\right) .
$$

The weights of $\mathbf{1 6}$ are $\frac{1}{2}\left\{\epsilon_{1}, \ldots, \epsilon_{8}\right\}$ where the $\epsilon$ 's can assume all possible signs; this means that $\mathbf{1 6}$ breaks into the direct sum of the conjugate irreducible spinor representations $\boldsymbol{8}_{s}$ and $\boldsymbol{8}_{c}$ of $S O(8)$, with $\frac{1}{2} C_{U(1)^{\prime \prime \prime}}$ measuring their chirality:

$$
\mathbf{1 6}=\mathbf{8}_{s,-1}+\mathbf{8}_{c, 1}
$$

which allows one to recover (4.43).

\subsubsection{A second chain of embeddings}

A second chain of maximal and symmetric embeddings, relevant in order to highlight the relation to the symmetry groups of the rank-3 Euclidean Jordan algebra $\mathfrak{J}_{3}(\mathbb{O})$ and also for a subsequent generalization at least for all conformal non-compact form of non-degenerate [64] groups of type $E_{7}$ [14] (see Section 5), reads as follows:

$$
\begin{aligned}
E_{7(-25)} & \supset E_{6(-26)} \times S O(1,1)^{\prime} \\
& \supset F_{4(-52)} \times S O(1,1)^{\prime} \\
& \supset S O(9) \times S O(1,1)^{\prime} \\
& \supset S O(8) \times S O(1,1)^{\prime},
\end{aligned}
$$

where $S O(8)$ in the fourth line of (4.53) coincides with the $S O(8)$ in the third line of (4.26) and (4.27). Moreover, (4.53) also clarifies (4.25). As 
already mentioned above, it holds that (see, e.g., $[29,30,65])$ :

$$
\begin{aligned}
& E_{7(-25)}=\operatorname{Conf}\left(\mathfrak{J}_{3}(\mathbb{O})\right)=\operatorname{Aut}\left[\mathfrak{M}\left(\mathfrak{J}_{3}(\mathbb{O})\right)\right]=G_{4} ; \\
& E_{6(-26)}=\operatorname{Str}_{0}\left(\mathfrak{J}_{3}(\mathbb{O})\right)=G_{5} ; \\
& F_{4(-52)}=\operatorname{Aut}\left(\mathfrak{J}_{3}(\mathbb{O})\right)=\operatorname{mcs}\left[\operatorname{Str}_{0}\left(\mathfrak{J}_{3}(\mathbb{O})\right)\right] ; \\
& \mathfrak{s o}(8)=\mathfrak{A} \mathfrak{u t}(\mathbf{t}(\mathbb{O}))=: \mathfrak{t r i}(O),
\end{aligned}
$$

where $\mathbf{t}(\mathbb{O})$ denotes the normed triality over the octonions $\mathbb{O}$ (see, e.g., [62]), and (4.13) has been recalled. In $(4.53), S O(1,1)$ has the physical meaning of "extra" $T$-duality generated by the Kaluza-Klein reduction $D=5 \rightarrow D=4$.

Correspondingly, the adjoint irrep. 133 of $E_{7(-25)}$ branches as (see, e.g., [32]):

$$
\begin{aligned}
& \mathbf{1 3 3} \rightarrow \mathbf{7 8 _ { 0 }}+\mathbf{1}_{0}+\mathbf{2 7 _ { - 2 }}+\mathbf{2 7 _ { + 2 } ^ { \prime }} \\
& \rightarrow \mathbf{2 6} \mathbf{6}_{0}+\mathbf{5} \mathbf{2}_{0}+\mathbf{1}_{0}+\mathbf{1}_{-2}+\mathbf{2 6}_{-2}+\mathbf{1}_{+2}+\mathbf{2 6}_{+2} \\
& \rightarrow 1_{0}+\mathbf{9}_{0}+16_{0}+16_{0}+36_{0}+1_{0} \\
& +\mathbf{1}_{-2}+\mathbf{1}_{-2}+\mathbf{9}_{-2}+\mathbf{1 6}_{-2} \\
& +\mathbf{1}_{+2}+\mathbf{1}_{+2}+\mathbf{9}_{+2}+\mathbf{1 6}_{+2} \\
& \rightarrow \mathbf{1}_{0}+\mathbf{1}_{0}+\mathbf{8}_{v, 0}+\mathbf{8}_{c, 0}+\mathbf{8}_{s, 0}+\mathbf{8}_{c, 0}+\mathbf{8}_{s, 0}+\mathbf{8}_{v, 0}+\mathbf{2 8}_{0}+\mathbf{1}_{0} \\
& +\mathbf{1}_{-2}+\mathbf{1}_{-2}+\mathbf{1}_{-2}+\mathbf{8}_{v,-2}+\mathbf{8}_{c,-2}+\mathbf{8}_{s,-2} \\
& +\mathbf{1}_{+2}+\mathbf{1}_{+2}+\mathbf{1}_{+2}+\mathbf{8}_{v,+2}+\mathbf{8}_{c,+2}+\mathbf{8}_{s,+2} \text {. }
\end{aligned}
$$

Analogously, the symplectic fundamental irrep. $\mathbf{5 6}$ of $E_{7(-25)}$ branches as (see, e.g., [32]):

$$
\begin{aligned}
\mathbf{5 6} & \rightarrow \mathbf{2 7}_{+1}+\mathbf{2 7}_{-1}^{\prime}+\mathbf{1}_{+3}+\mathbf{1}_{-3} \\
& \rightarrow \mathbf{1}_{+1}+\mathbf{2 6}_{+1}+\mathbf{1}_{-1}+\mathbf{2 6}_{-1}+\mathbf{1}_{+3}+\mathbf{1}_{-3} \\
& \rightarrow \mathbf{1}_{+1}+\mathbf{1}_{+1}+\mathbf{9}_{+1}+\mathbf{1 6}_{+1}+\mathbf{1}_{-1}+\mathbf{1}_{-1}+\mathbf{9}_{-1}+\mathbf{1 6}_{-1}+\mathbf{1}_{+3}+\mathbf{1}_{-3} \\
& \rightarrow \mathbf{1}_{+1}+\mathbf{1}_{+1}+\mathbf{1}_{+1}+\mathbf{8}_{v,+1}+\mathbf{8}_{c,+1}+\mathbf{8}_{s,+1} \\
& +\mathbf{1}_{-1}+\mathbf{1}_{-1}+\mathbf{1}_{-1}+\mathbf{8}_{v,-1}+\mathbf{8}_{c,-1}+\mathbf{8}_{s,-1}+\mathbf{1}_{+3}+\mathbf{1}_{-3} .
\end{aligned}
$$

\subsubsection{Comments on cartan subalgebras}

In the analysis made in Sections 4.2.1 and 4.2.2, the 3-dimensional noncompact Cartan subalgebra $\mathfrak{H}_{3}$ (4.1) of $\mathcal{M}(1.2)$ is generated by a suitable 
linear combination of the six $S O(8)$-singlets (4.39). Thus, $\mathfrak{H}_{3}$ is not the Lie algebra of the group factor

$$
\widetilde{H}_{3}:=[S O(1,1)]^{3} \subsetneq E_{6(-26)} \times S O(1,1)
$$

commuting with $S O(8)$ in the branching (4.27), because by definition for the Lie group $H_{3}$ generated by $\mathfrak{H}_{3}$ it holds that (recall definition (3.20)):

$$
H_{3} \subsetneq \frac{E_{7(-25)}}{E_{6(-26)} \times S O(1,1)}=: \widetilde{\mathcal{M}}
$$

and, by definition:

$$
\mathfrak{T} \cap \mathfrak{P}=0 .
$$

As stated in the previous treatment, $\mathfrak{H}_{3}$ can be extended to a 7 -dimensional maximal Cartan subalgebra $\mathfrak{H}$ of $\mathfrak{e}_{7(-25)}$ by adding a 4-dimensional maximal Cartan subalgebra of $\mathfrak{s o}(8)$, which is clearly compact:

$$
\mathfrak{H}_{7}:=\mathfrak{H}_{3} \oplus \mathfrak{H}_{4},
$$

with signature $\left(++^{3},-^{4}\right)$ (indeed, as mentioned above, compact generators are conventionally chosen with negative signature).

On the other hand, the factor $\widetilde{H}_{3} \equiv[S O(1,1)]^{3}$ in (4.60) which commutes with $S O(8)$ in the branching $(4.27)$ can be extended to $[S L(2, \mathbb{R})]^{3}$, which with further branchings gives rise to the (not maximal nor symmetric) embedding:

$$
E_{6(-26)} \supsetneq[S U(2)]^{4} \times[S L(2, \mathbb{R})]^{3},
$$

recently considered in [7] within the quantum-informational interpretation of $\mathcal{N}=2, D=4$ exceptional magic supergravity. As done above for $\mathfrak{H}_{3}$, the Lie algebra $\widetilde{\mathfrak{H}}_{3}$ of $\widetilde{H}_{3}$ can be extended to another 7-dimensional maximal Cartan subalgebra $\widetilde{\mathfrak{H}}_{7}$ of $\mathfrak{e}_{7(-25)}$ by adding a 4-dimensional maximal Cartan subalgebra of $\mathfrak{s o}(8)$, which is clearly compact:

$$
\widetilde{\mathfrak{H}}_{7}:=\widetilde{\mathfrak{H}}_{3} \oplus \mathfrak{H}_{4}
$$

once again with signature $\left(+{ }^{3},-{ }^{4}\right)$. 


\section{Generalizations to groups of type $\mathrm{E}_{7}$}

The results derived until now hold at least for the conformal non-compact real forms of (non-degenerate [64]) simple groups "of type $E_{7}$ " [14,15, 19, 20]. The first axiomatic characterization of groups "of type $E_{7}$ " through a module (irreducible representation) was given in 1967 by Brown [14]. A group $G$ "of type $E_{7}$ " is a Lie group endowed with a representation $\mathbf{R}$ such that:

- $\mathbf{R}$ is symplectic, i.e., (the subscripts " $s$ " and " $a$ " stand for symmetric and skew-symmetric throughout):

$$
\exists ! \mathbb{C}_{[M N]} \equiv \mathbf{1} \in \mathbf{R} \times{ }_{a} \mathbf{R}
$$

$\mathbb{C}_{[M N]}$ defines a non-degenerate skew-symmetric bilinear form (symplectic product); given two different charge vectors $\mathcal{Q}_{x}$ and $\mathcal{Q}_{y}$ in $\mathbf{R}$, such a bilinear form is defined as:

$$
\left\langle\mathcal{Q}_{x}, \mathcal{Q}_{y}\right\rangle \equiv \mathcal{Q}_{x}^{M} \mathcal{Q}_{y}^{N} \mathbb{C}_{M N}=-\left\langle\mathcal{Q}_{y}, \mathcal{Q}_{x}\right\rangle
$$

- $\mathbf{R}$ admits a unique rank-4 completely symmetric primitive $G$-invariant structure, usually named $K$-tensor:

$$
\exists ! \mathbb{K}_{(M N P Q)} \equiv \mathbf{1} \in[\mathbf{R} \times \mathbf{R} \times \mathbf{R} \times \mathbf{R}]_{s}
$$

thus, by contracting the $K$-tensor with the same charge vector $\mathcal{Q}$ in $\mathbf{R}$, one can construct a rank- 4 homogeneous $G$-invariant polynomial (whose $\varsigma$ is the normalization constant):

$$
\mathbf{q}(\mathcal{Q}) \equiv \varsigma \mathbb{K}_{M N P Q} \mathcal{Q}^{M} \mathcal{Q}^{N} \mathcal{Q}^{P} \mathcal{Q}^{Q}
$$

which corresponds to the evaluation of the rank- 4 symmetric invariant q-structure induced by the $K$-tensor on four identical modules $\mathbf{R}$ :

$$
\begin{aligned}
\mathbf{q}(Q) & \left.\equiv \mathbf{q}\left(\mathcal{Q}_{x}, \mathcal{Q}_{y}, \mathcal{Q}_{z}, \mathcal{Q}_{w}\right)\right|_{\mathcal{Q}_{x}=\mathcal{Q}_{y}=\mathcal{Q}_{z}=\mathcal{Q}_{w} \equiv \mathcal{Q}} \\
& \equiv \varsigma\left[\mathbb{K}_{M N P Q} \mathcal{Q}_{x}^{M} \mathcal{Q}_{y}^{N} \mathcal{Q}_{z}^{P} \mathcal{Q}_{w}^{Q}\right]_{\mathcal{Q}_{x}=\mathcal{Q}_{y}=\mathcal{Q}_{z}=\mathcal{Q}_{w} \equiv \mathcal{Q}}
\end{aligned}
$$

A famous example of quartic invariant in $G=E_{7}$ is the CartanCremmer-Julia invariant ${ }^{4}$ ( [68], p. 274), constructed out of the fundamental representation $\mathbf{R}=\mathbf{5 6}$.

\footnotetext{
${ }^{4}$ As also mentioned in [66], it should be noted that the quartic form is given incorrectly by Cartan; the error seems to have been first observed by Freudenthal [67].
} 
- If a trilinear map $T: \mathbf{R} \times \mathbf{R} \times \mathbf{R} \rightarrow \mathbf{R}$ is defined such that:

$$
\left\langle T\left(\mathcal{Q}_{x}, \mathcal{Q}_{y}, \mathcal{Q}_{z}\right), \mathcal{Q}_{w}\right\rangle=\mathbf{q}\left(\mathcal{Q}_{x}, \mathcal{Q}_{y}, \mathcal{Q}_{z}, \mathcal{Q}_{w}\right),
$$

then it holds that:

$$
\left\langle T\left(\mathcal{Q}_{x}, \mathcal{Q}_{x}, \mathcal{Q}_{y}\right), T\left(\mathcal{Q}_{y}, \mathcal{Q}_{y}, \mathcal{Q}_{y}\right)\right\rangle=-2\left\langle\mathcal{Q}_{x}, \mathcal{Q}_{y}\right\rangle \mathbf{q}\left(\mathcal{Q}_{x}, \mathcal{Q}_{y}, \mathcal{Q}_{y}, \mathcal{Q}_{y}\right)
$$

This last property makes the group of type $E_{7}$ amenable to a treatment in terms of (rank-3) Jordan algebras and related Freudenthal triple systems.

Remarkably, groups of type $E_{7}$, appearing in $D=4$ supergravity as $U$ duality groups, admit a $D=5$ uplift to groups of type $E_{6}$, as well as a $D=3$ downlift to groups of type $E_{8}$. It should also be recalled that split forms of exceptional Lie groups of type $E$ appear in the exceptional Cremmer-Julia [39] sequence $E_{11-D,(11-D)}$ of $U$-duality groups of $M$-theory compactified on a $D$-dimensional torus, in $D=3,4,5$. Other sequences, composed by non-split, non-compact real forms of exceptional groups, are also relevant to non-maximal supergravity in various dimensions (see, e.g., the treatment in [61], also for a list of related references).

The connection of groups of type $E_{7}$ to supergravity can be summarized by stating that all $2 \leqslant \mathcal{N} \leqslant 8$-extended supergravities in $D=4$ with symmetric scalar manifolds $\frac{G_{4}}{H_{4}}$ have $G_{4}$ of type $E_{7}[15,19]$. It is intriguing to notice that the first paper on groups of type $E_{7}$ was written about a decade before the discovery of of extended $(\mathcal{N}=2)$ supergravity [69], in which electromagnetic duality symmetry was observed [70].

In particular, simple $U$-duality groups of $\mathcal{N}=2, D=4$ theories with symmetric (vector multiplets') scalar manifolds (listed in Table 3) are conformal non-compact, real forms of simple non-degenerate groups of type $E_{7}$, which are the conformal symmetry group of simple Euclidean Jordan algebras of rank $3[25]$.

Furthermore, the results of Sections 2 and 3 also hold for the relevant non-compact, real forms of (non-degenerate [64]) semi-simple groups of type $E_{7}[14,15,19,20]$, appearing in supergravity as semi-simple $U$ - duality group of the infinite sequence of $\mathcal{N}=2$ theories, with scalar manifold given by:

$$
\frac{S L(2, \mathbb{R})}{U(1)} \times \frac{S O(2, n)}{S O(2) \times S O(n)}, n \geqslant 1, \quad \operatorname{rank}=1+\min (2, n),
$$

based on the semi-simple rank-3 Jordan algebra $\mathbb{R} \oplus \boldsymbol{\Gamma}_{1, n-1}$, where $\boldsymbol{\Gamma}_{1, n-1}$ stands for the Jordan algebra of degree two with a quadratic form of 
Table 3: Vector multiplets' symmetric scalar manifolds (5.9) (up to possible finite factors in the stabiliser) of $\mathcal{N}=2, D=4$ supergravity models with simple $U$-duality groups (alias conformal non-compact real forms of nondegenerate [64], simple group of type $E_{7}[14,15,19,20]$ ), with related simple rank-3 Jordan algebra. The relevant symplectic irrep. $\mathbf{R}$ of $G_{4}$ is also reported. $\mathbb{O}, \mathbb{H}, \mathbb{C}$, and $\mathbb{R}$, respectively denote the four division algebras of octonions, quaternions, complex, and real numbers. Note that, with the exception of the triality symmetric STU model [53], these models are all the ones for which the treatment of [61] holds (see, e.g., Table 1 therein). The $D=5$ uplift of the $T^{3}$ model based on $\mathfrak{J}_{3}=\mathbb{R}$ is the pure $\mathcal{N}=2, D=5$ supergravity. $\mathfrak{J}_{3}(\mathbb{H})$ is related to both 8 and 24 supersymmetries, because the corresponding supergravity theories are "twin", namely they share the very same bosonic sector [72].

\begin{tabular}{lccc}
\hline $\mathfrak{J}_{3}$ & $G_{4} / H_{4}$ & $\mathbf{R}$ & $q$ \\
\hline $\mathfrak{J}_{3}(\mathbb{O})$ & $\frac{E_{7(-25)}}{E_{6(-78)} \times U(1)}$ & $\mathbf{5 6}$ & 8 \\
$\mathfrak{J}_{3}(\mathbb{H})$ & $\frac{S O^{*}(12)}{S U(6) \times U(1)}$ & $\mathbf{3 2}$ & 4 \\
$\mathfrak{J}_{3}(\mathbb{C})$ & $\frac{S U(3,3)}{S U(3) \times S U(3) \times U(1)}$ & $\mathbf{2 0}$ & 2 \\
$\mathfrak{J}_{3}(\mathbb{R})$ & $\frac{S p(6, \mathbb{R})}{S U(3) \times U(1)}$ & $\mathbf{1 4}$ & 1 \\
$\mathbb{R}\left(T^{3}\right.$ model $)$ & $\frac{S L(2, \mathbb{R})}{U(1)}$ & $\mathbf{4}$ & $-2 / 3$ \\
\hline
\end{tabular}

Lorentzian signature $(1, n-1)$, which is nothing but the Clifford algebra of $O(1, n-1)$ [71].

In other words, at the group level, the results of Sections 2 and 3 provide a manifestly $\left[\operatorname{mcs}\left(\operatorname{Conf}\left(\mathfrak{J}_{3}\right)\right)\right]$-covariant symplectic frame for the special Kähler geometry of the corresponding symmetric, non-compact, vector multiplet's scalar manifold (of Riemannian nature), whose coset structure reads (up to possible finite factors in the stabilizer; see, e.g., [29,65]; for a comprehensive list of manifolds, see, e.g., [73]):

$$
\mathcal{M}_{\mathcal{N}=2}=\frac{\operatorname{Conf}\left(\mathfrak{J}_{3}\right)}{\operatorname{mcs}\left(\operatorname{Conf}\left(\mathfrak{J}_{3}\right)\right)} .
$$

Here $\operatorname{Conf}\left(\mathfrak{J}_{3}\right)=\operatorname{Aut}\left(\mathfrak{M}\left(\mathfrak{J}_{3}\right)\right)$ stands for the conformal group of $\mathfrak{J}_{3}$, which is nothing but the automorphism group of the Freudenthal triple system 
$\mathfrak{M}[28]$ constructed on $\mathfrak{J}_{3}$ itself. The relevant non-compact, real forms of $\operatorname{mcs}\left[\operatorname{Conf}\left(\mathfrak{J}_{3}\right)\right] / U(1)$ (namely, the $U$-duality symmetries in $D=5$ ) are the reduced structure algebras of the corresponding ( $q$-parametrized) simple, rank-3 Euclidean Jordan algebras.

Up to symplectic re-parametrization, for the infinite sequence (5.8) of $\mathcal{N}=2$ theories with semi-simple $U$-duality group $\operatorname{Conf}\left(\mathfrak{J}_{3}\right)=S L(2, \mathbb{R}) \times$ $S O(2, n)$, the results of Sections 2 and 3 match the so-called Calabi-Vesentini $\mathcal{N}=2$ symplectic frame [57,74] (see also [20] for a recent study), whose (compact) manifest covariance is the maximal one:

$$
\operatorname{mcs}\left(\operatorname{Conf}\left(\mathfrak{J}_{3}\right)\right)=\operatorname{mcs}(S L(2, \mathbb{R}) \times S O(2, n))=U(1) \times S O(2) \times S O(n) .
$$

All the vector multiplets' scalar manifolds of the aforementioned $\mathcal{N}=$ 2, $D=4$ supergravity theories related to cubic Euclidean Jordan algebras are special Kähler, maximal, non-compact, symmetric cosets with structure (5.9), and have rank $^{5} 3$ (except the rank-1 case of $T^{3}$ model). They also are Einstein spaces, with constant (negative) Ricci scalar curvature $R$ :

$$
R_{i \bar{j}}=\lambda g_{i \bar{j}} \Rightarrow R=\lambda n_{V}
$$

where $R_{i \bar{j}}$ is the special Kähler Ricci tensor, and the real parameter $\lambda$ has been computed in [76] (see also [51]):

$$
\lambda=\left\{\begin{array}{l}
-\frac{2}{3} n_{V} \text { for: } T^{3} \text { model }\left(n_{V}=1\right), S T U \text { model }\left(n_{V}=3\right), \\
\quad \text { and } \mathfrak{J}_{3}(\mathbb{A}) \text {-models }\left(n_{V}=3 q+3\right) ; \\
-\frac{\left(n_{V}^{2}-2 n_{V}+3\right)}{n_{V}} \text { for } \mathbb{R} \oplus \boldsymbol{\Gamma}_{1, n-1} \text { models }\left(n_{V}=n+1 \geqslant 2\right) .
\end{array}\right.
$$

Similarly, also the results about the Iwasawa decomposition worked out in Section 4 can be generalized at least to the conformal non-compact real forms of (non-degenerate [64]) simple groups of type $E_{7}[14,15,19,20]$, listed as $D=4 U$-duality groups $G_{4}$ 's in Table 3 .

\footnotetext{
${ }^{5}$ The rank of a manifold is defined as the maximal dimension (in $\mathbb{R}$ ) of a Riemann-flat, totally geodesic sub-manifold of the manifold itself (see, e.g., [75], p. 209).
} 
Indeed, in light of (4.54)-(4.57), the chain of maximal and symmetric embeddings (4.53) enjoys the following generalization:

$$
\begin{aligned}
\operatorname{Conf}\left(\mathfrak{J}_{3}(\mathbb{A})\right) & \supset \operatorname{Str}_{0}\left(\mathfrak{J}_{3}(\mathbb{A})\right) \times S O(1,1)^{\prime} \\
& \supset \operatorname{Aut}\left(\mathfrak{J}_{3}(\mathbb{A})\right) \times S O(1,1)^{\prime} \\
& \supset S O(q+1) \times \mathcal{A}_{q} \times S O(1,1)^{\prime} \\
& \supset S O(q) \times \mathcal{A}_{q} \times S O(1,1)^{\prime},
\end{aligned}
$$

by recalling the definition introduced just below $(2.30), q:=\operatorname{dim}_{\mathbb{R}} \mathbb{A}=1,2,4$, and 8 for $\mathbb{A}=\mathbb{R}, \mathbb{C}, \mathbb{H}$, and $\mathbb{O}$, respectively. We report the symmetry groups of simple rank-3 Euclidean Jordan algebras in Table 4. Mutatis mutandis (also with the help of the tables), the treatment and the results of the whole Section 4 can be extended to all $\mathcal{N}=2, D=4$ symmetric supergravities reported in Table 3 (but the $T^{3}$ model).

The extra factor group $\mathcal{A}_{q}$, which exists only for $q=2$ and $q=4$, is reported in Table 5; in [77], its appearance was observed within the study of the charge orbits of asymptotically flat 0-(black holes) and 1-(black strings) branes in minimal magical Maxwell-Einstein supergravity theories in $D=5$ space-time dimensions. We note that $\mathcal{A}_{q}$ is related to $\widehat{G}_{\text {cent }}$ and $G_{\text {paint }}$ (Lie groups usually introduced in the treatment of supergravity billiards and timelike Kaluza-Klein reductions; for recent treatment and set of related references, see, e.g., [58]; see also Table 5 therein, also for subtleties concerning the case $q=8$ in $D=5,6)$ as follows [77]:

$$
\begin{aligned}
& D=5,6: \widehat{G}_{\text {cent }}=S O(1,1) \times S O(q-1) \times \mathcal{A}_{q} ; \\
& D=3,4: \widehat{G}_{\text {cent }}=G_{\text {paint }}=S O(q) \times \mathcal{A}_{q} .
\end{aligned}
$$

According to [78], $\mathcal{A}_{q}$ can be related to the structure of the Hopf maps, chiral Weyl spinors and division algebras; we hope to study this intriguing connection in future investigations, also along the lines of [79].

Extending the considerations made above on $S O(8)$, it can be observed that $S O(q) \times \mathcal{A}_{q}$ shares the same Lie algebra $\operatorname{tri}(\mathbb{A})$ of $\operatorname{Aut}(\mathbf{t}(\mathbb{A}))$, which is the automorphism group of the normed triality over the division algebra $\mathbb{A}$ (see, e.g., [62]); see Table 6.

Besides this fact, it is intriguing to notice that $S O(q) \times \mathcal{A}_{q}$ appears in at least three (apparently unrelated) contexts:

(1) As $\widehat{G}_{\text {cent }}=G_{\text {paint }}$ in $D=3,4$, as given by $(5.15)$ (see, e.g., $[58,77]$ ). 


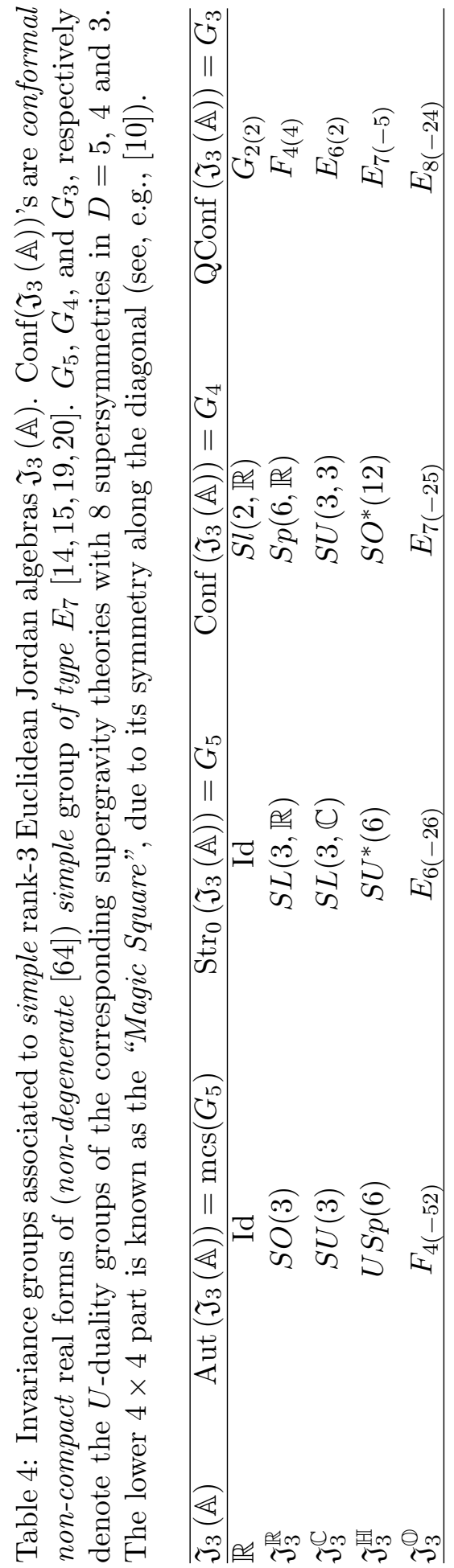


Table 5: The extra commuting group $\mathcal{A}_{q}$ (see, e.g., [77]).

\begin{tabular}{cc}
\hline$q$ & $\mathcal{A}_{q}$ \\
\hline 8 & - \\
4 & $S O(3)$ \\
2 & $S O(2)$ \\
1 & - \\
\hline
\end{tabular}

(2) As stabilizer group $\mathcal{G}_{p=2}\left(\mathfrak{J}_{3}(\mathbb{A})\right)$ of BPS generic charge orbits of 2centered extremal black holes in $\mathcal{N}=2, D=4$ magical models, as derived in [17], and reported in Table 7.

(3) According to (5.13), as group of maximal manifest covariance of the Iwasawa decomposition of the (vector multiplets') scalar manifold of $\mathcal{N}=2, D=4$ magical models, whose $U$-duality groups are (some instances of) conformal non-compact real forms of (non-degenerate [64]) simple groups of type $E_{7}[14,15,19,20]$.

\section{Conclusion}

The present investigation, and in particular the generalizations discussed in Section 5, pave the way to a number of interesting further developments. We list a selection of them below.

Starting with the treatment given in Section 3, it should be pointed out that a more explicit expression of the symplectic frame determined by the comparison of (3.7) and (3.8) with the general formulae (3.10)-(3.14) of special Kähler geometry would be needed also in order to check that the prepotential $F$ does not exist in the symplectic frame introduced in Sections 2 and 3, which can be considered the analogue of the Calabi-Vesentini one $[57,74]$ for non-degenerate, conformal non-compact, and simple groups of type $E_{7}$.

Furthermore, it would be interesting to extend the maximally manifestlycovariant symplectic frame and/or the Iwasawa symplectic frame, respectively introduced in Sections 2, 3 and in Section 4, to

- compact groups of type $E_{7}$;

- other non-compact real forms of groups of type $E_{7}$ (possibly related to $\mathcal{N}>2$-extended supergravity theories), also in relation to rank3 Jordan algebras on split forms of division algebras (for a recent treatment of the non-supersymmetric cases of $\mathfrak{J}_{3}\left(\mathbb{H}_{s}\right)$ and $\mathfrak{J}_{3}\left(\mathbb{C}_{s}\right)$, see, e.g., [30]); 


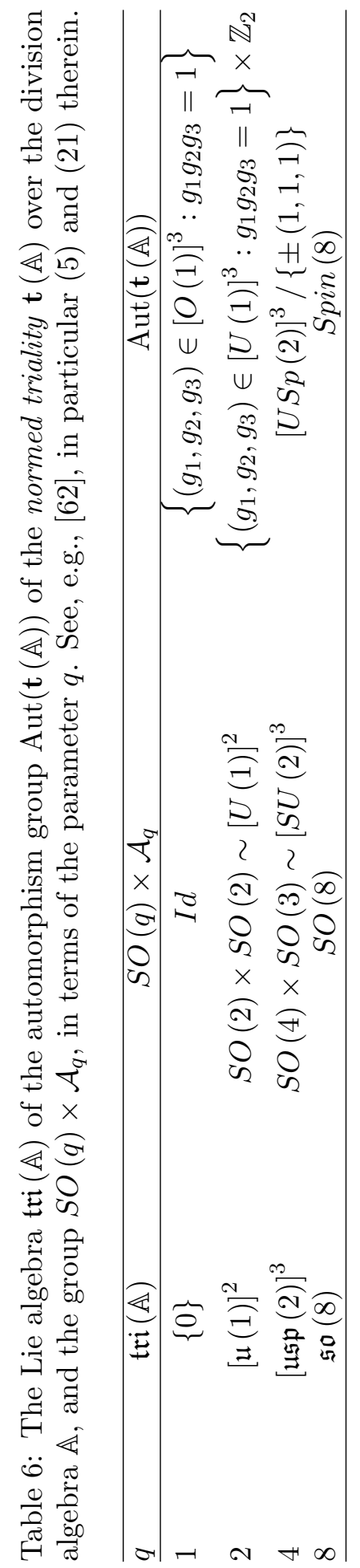


Table 7: BPS generic charge orbits of 2-centered extremal black holes in $\mathcal{N}=2, d=4$ magical models. $\operatorname{Conf}\left(\mathfrak{J}_{3}(\mathbb{A})\right)$ denotes the "conformal" group of $\mathfrak{J}_{3}(\mathbb{A})$ (see, e.g., [65]) [17].

\begin{tabular}{lc}
\hline$A$ & $\mathcal{O}_{p=2, B P S}=\frac{\mathfrak{J}_{3}(\mathbb{A})}{\mathcal{G}_{p=2}\left(\mathfrak{J}_{3}(\mathbb{A})\right)}$ \\
$\mathbb{1}$ & $\frac{E_{7(-25)}}{S O(8)}$ \\
$\mathbb{H}$ & $\frac{S O^{*}(12)}{[S U(2)]^{3}}$ \\
$\mathbb{C}$ & $\frac{S U(3,3)}{[U(1)]^{2}}$ \\
$\mathbb{R}$ & $S p(6, \mathbb{R})$ \\
\hline
\end{tabular}

- other classes of supergravities, such as $\mathcal{N}=2, D=4$ with homogeneous non-symmetric scalar manifolds [37,41].

Furthermore, considering the generalizations of the Iwasawa parametrization discussed in Section 5, it would be interesting to explore its extension also to theories related to semi-simple rank-3 Jordan algebras, such as $\mathbb{R} \oplus \boldsymbol{\Gamma}_{m, n}$ (for $m=1$, recall (5.8)), where $\boldsymbol{\Gamma}_{m, n}$ stands for the rank-2 Jordan algebra with a quadratic form of Lorentzian signature $(m, n)$, which is nothing but the Clifford algebra of $O(m, n)$ [71].

Concerning the generalization to $\mathcal{N}>2$-extended supergravities, it would be interesting to compare the application of the Iwasawa decomposition under consideration to the case of $\mathbb{O}_{S}$ with the Iwasawa parametrization of $\mathcal{M}_{\mathcal{N}=8}$ (3.9) studied in [22], whose manifest maximal (non-compact) covariance is $S L(7, \mathbb{R})$, with mcs $S O(7)$. In this respect, the following remark made in [17] should be relevant: as it holds for the stabilizer of $\mathcal{O}_{\mathcal{N}=2, \widetilde{J}_{3}(\mathbb{Q}), \operatorname{tab} 7, p=2}($ see Table 7$)$, the Lie algebra $\mathfrak{s o}(8)$ of the stabilizer of the 2-centered orbit [17]

$$
\mathcal{O}_{\mathcal{N}=2, J_{3}^{\oplus}, \mathrm{nBPS}, p=2, \mathbf{I}}=\frac{E_{7(-25)}}{S O(8)}
$$

is nothing but the Lie algebra $\operatorname{tri}(\mathbb{O})$ of the automorphism group Aut $(\mathbf{t}(\mathbb{O})$ ) of the normed triality over $\mathbb{O}$ (see Table 6 ). It is here worth observing that the Lie algebra $\mathfrak{s o}(4,4)$ of the stabilizer of the 2-centered orbit [17]

$$
\mathcal{O}_{\mathcal{N}=8, \frac{1}{8}-\mathrm{BPS}, p=2, \mathbf{I}}=\frac{E_{7(7)}}{S O(4,4)}
$$


enjoys an analogous interpretation as the Lie algebra $\mathfrak{t r i}\left(\mathbb{O}_{s}\right)$ of the automorphism group $\operatorname{Aut}\left(\mathbf{t}\left(\mathbb{O}_{S}\right)\right)$ of the normed triality over $\mathbb{O}_{S}$. On the other hand, a similar interpretation does not seem to hold for the stabilizer of the 2-centered orbit [17]

$$
\mathcal{O}_{\mathcal{N}=8, \frac{1}{8}-\mathrm{BPS}, p=2, \mathbf{I I}}=\frac{E_{7(7)}}{S O(5,3)},
$$

as well as for the stabilizer of the 2-centered orbit [17]

$$
\mathcal{O}_{\mathcal{N}=2, J_{3}^{\oplus}, \mathrm{nBPS}, p=2, \mathbf{I I}}=\frac{E_{7(-25)}}{S O(7,1)} .
$$

However, it is intriguing to note that the maximal manifest compact covariance $S O(7)=\operatorname{mcs}(S O(7,1))=\operatorname{mcs}(S L(7, \mathbb{R}))$ exhibited by the Iwasawa parametrization of $\mathcal{M}_{\mathcal{N}=8}$ (3.9) [22] may provide a clue for the stabilizer of $\mathcal{O}_{\mathcal{N}=2, J_{3}^{\oplus}, \text { nBPS, } p=2, \text { II }}$ (6.4). We leave to future studies the in-depth investigation of these fascinating connections, here just briefly outlined.

Finally, we would like to put forward an hint ${ }^{6}$ for a further physical application. It should be observed that the Iwasawa coset decomposition can yield a nilpotent algebra exhibiting the same symmetry of the systems recently discussed in [80] in the framework of supergravity theories timelike-reduced down to $D=3^{*}$ dimensions. Thus, it would be interesting to investigate the possible Lax pair structures hidden in the Iwasawa formalism, which might allow for a more explicit integration procedure within the $D=3^{*}$ nilpotent orbits formalism of [80]. We hope to report on this intriguing connection in future studies.

\section{Acknowledgments}

We would like to thank Leron Borsten, Sergio Ferrara and Augusto Sagnotti for enlightening discussions. We also thank Francesco Dalla Piazza for technical support. The work of B.L.C. has been supported in part by the European Commission under the FP7-PEOPLE-IRG-2008 Grant No. PIRG04-GA-2008-239412 "String Theory and Noncommutative Geometry" (STRING).

\footnotetext{
${ }^{6}$ We are grateful to Augusto Sagnotti for an enlightening remark on this.
} 


\section{References}

[1] P. Ramond, Exceptional Groups and Physics, Plenary Talk delivered at the Conference Groupe 24, Paris, July 2002, arXiv:hep-th/ 0301050v1.

[2] R. Coldea, D.A. Tennant, E.M. Wheeler, E. Wawrzynska, D. Prabhakaran, M. Telling, K. Habicht, P. Smeibidl and K. Kiefer, Quantum Criticality in an Ising Chain: Experimental Evidence for Emergent $E_{8}$ Symmetry, Science 327(5962) (2010), 177.

[3] D. Borthwick and S. Garibaldi, Did a 1-Dimensional Magnet Detect a 248-Dimensional Lie Algebra?, Not. Amer. Math. Soc. 58(8) (2011), 1055.

[4] J. Braun, A. Eichhorn, H. Gies and J.M. Pawlowski, On the Nature of the Phase Transition in $S U(N)$, Sp(2) and E(7) Yang-Mills theory, Eur. Phys. J. C 70 (2010), 689; arXiv:1007.2619 [hep-ph].

[5] M.J. Duff, String Triality, Black Hole Entropy and Cayley's Hyperdeterminant, Phys. Rev. D 76 (2007), 025017; arXiv: hep-th/0601134. M.J. Duff and S. Ferrara, $E_{7}$ and the Tripartite Entanglement of Seven Qubits, Phys. Rev. D 76 (2007), 025018; arXiv:quant-ph/0609227.

P. Levay, Stringy Black Holes and the Geometry of Entanglement, Phys. Rev. D 74 (2006), 024030; arXiv: hep-th/0603136.

[6] L. Borsten, D. Dahanayake, M.J. Duff, A. Marrani and W. Rubens, Four-Qubit Entanglement from String Theory, Phys. Rev. Lett. 105 (2010), 100507; arXiv:1005.4915 [hep-th].

[7] L. Borsten, M.J. Duff, A. Marrani and W. Rubens, On the BlackHole/Qubit Correspondence, Eur. Phys. J. Plus 126 (2011), 37; arXiv:1101.3559v1 [hep-th].

[8] B.L. Cerchiai and B. Van Geemen, From Qubits to $E_{7}$, J. Math. Phys. 51 (2010), 122203; arXiv:1003.4255 [quant-ph].

[9] S. Ferrara, R. Kallosh and A. Strominger, N=2 extremal black holes, Phys. Rev. D 52 (1995), 5412.

A. Strominger, Macroscopic entropy of $N=2$ extremal black holes, Phys. Lett. B 383 (1996), 39.

S. Ferrara and R. Kallosh, Supersymmetry and attractors, Phys. Rev.

D 54 (1996), 1514.

S. Ferrara and R. Kallosh, Universality of supersymmetric attractors, Phys. Rev. D 54 (1996), 1525.

S. Ferrara, G. W. Gibbons and R. Kallosh, Black holes and critical points in moduli space, Nucl. Phys. B 500 (1997), 75 (1997).

[10] M. Günaydin, G. Sierra, P.K. Townsend, Exceptional supergravity theories and the magic square, Phys. Lett. B 133B (1983), 72. 
M. Günaydin, G. Sierra and P.K. Townsend, The Geometry of $\mathcal{N}=2$ Maxwell-Einstein supergravity and Jordan Algebras, Nucl. Phys. B 242 (1984), 244.

Gauging the $D=5$ Maxwell-Einstein supergravity theories: more on Jordan Algebras, Nucl. Phys. B 253 (1985), 573.

More on $D=5$ Maxwell-Einstein supergravity: symmetric space and kinks, Class. Quant. Grav. 3 (1986), 763.

[11] V.K. Dobrev, Exceptional lie algebra $E_{7(-25)}$ : multiplets and invariant differential operators, J. Phys. A 42 (2009), 285203; arXiv:0812.2690 [hep-th].

[12] S.L. Cacciatori, F.D. Piazza and A. Scotti, $E_{7}$ groups from octonionic magic square, arXiv:1007.4758 [math-ph].

[13] P. Truini, G. Olivieri and L.C. Biedenharn, The Jordan pair content of the magic square and the geometry of the scalars in $\mathcal{N}=2$ Supergravity, Lett. Math. Phys. 9 (1985), 255.

P. Truini, Scalar manifolds and Jordan pairs in supergravity, Int. J. Theor. Phys. 25 (1986), 509.

[14] R.B. Brown, Groups of type $E_{7}$, J. Reine Angew. Math. 236 (1969), 79.

[15] L. Borsten, D. Dahanayake, M.J. Duff and W. Rubens, Black holes admitting a Freudenthal dual, Phys. Rev. D 80 (2009), 026003; arXiv:0903.5517 [hep-th].

[16] S. Ferrara, A. Marrani, E. Orazi, R. Stora and A. Yeranyan, Two-center black holes duality-invariants for stu model and its lower-rank descendants, J. Math. Phys. 52 (2011), 062302; arXiv:1011.5864 [hep-th] .

[17] L. Andrianopoli, R. D'Auria, S. Ferrara, A. Marrani and M. Trigiante, Two-centered magical charge orbits, J. High Energy Phys. 04 (2011), 041; arXiv: 1101.3496 [hep-th].

[18] A. Ceresole, S. Ferrara, A. Marrani and A. Yeranyan, Small black hole constituents and horizontal symmetry, J. High Energy Phys. 06 (2011), 078; arXiv:1104.4652 [hep-th].

[19] S. Ferrara, A. Marrani and A. Yeranyan, Freudenthal duality and generalized special geometry, Phys. Lett. B 701 (2011), 640; arXiv: 1102.4857 [hep-th].

[20] S. Ferrara, A. Marrani and A. Yeranyan, On invariant structures of black hole charges, J. High Energy Phys. 12 (2012), 71; arXiv: 1110.4004 [hep-th].

[21] S. Ferrara and R. Kallosh, Creation of matter in the universe and groups of type $E_{7}$, J. High Energy Phys. 12 (2011), 096; arXiv:1110.4048 [hep-th]. 
[22] S.L. Cacciatori, B.L. Cerchiai and A. Marrani, Iwasawa $\mathcal{N}=8$ Attractors, J. Math. Phys. 51 (2010), 102502; arXiv:1005.2231 [hep-th].

[23] I.L. Kantor and A. S. Solodovnikov, Normed algebras with an identity. Hurwitz's theorem, in 'Hypercomplex Numbers. An Elementary Introduction to Algebras', Spinger-Verlag, 1989.

[24] I. Yokota, Exceptional lie groups, arXiv:0902.0431 [math.DG].

[25] C.H. Barton and A. Sudbery, Magic squares and matrix models of Lie algebras, Adv. Math. 180 (2003), 596; arXiv:math/0203010 [math.RA].

[26] J. Tits, Algèbres alternatives, algèbres de Jordan et algèbres de Lie exceptionnelles. I. Construction, (French), Nederl. Akad. Wetensch. Proc'Ser. A 69 (1966), 223.

[27] M. Günaydin and O. Pavlyk, Spectrum generating conformal and quasiconformal $U$-duality groups, supergravity and spherical vectors, J. High Energy Phys. 04 (2010), 070; arXiv:0901.1646 [hep-th].

[28] H. Freudenthal, Oktaven, Ausnahmegruppen und Oktavengeometrie, Geom. Dedicata 19 (1985), 7.

[29] L. Borsten, M.J. Duff, S. Ferrara, A. Marrani and W. Rubens, Small orbits, arXiv:1108.0424 [hep-th].

[30] L. Borsten, M.J. Duff, S. Ferrara, A. Marrani and W. Rubens, Explicit orbit classification of reducible Jordan algebras and freudenthal triple systems, arXiv:1108.0908 [math.RA].

[31] M. Günaydin and O. Pavlyk, Quasiconformal realizations of $E_{6(6)}$, $E_{7(7)}, E_{8(8)}$ and $S O(n+3, m+3), \mathcal{N} \geqslant 4$ supergravity and spherical vectors, arXiv:0904.0784 [hep-th].

[32] R. Slansky, Group theory for unified model building, Phys. Rep. 79 (1981), 1.

[33] W.G. McKay and J. Patera, Tables of dimensions, indices, and branching rules for representations of simple lie algebras, M. Dekker, New York, 1981.

[34] F. Bernardoni, S.L. Cacciatori, B.L. Cerchiai and A. Scotti, Mapping the geometry of the $F_{4}$ group, Adv. Theor. Math. Phys. 12(4) (2008), 889; arXiv:0705.3978 [math-ph].

[35] F. Bernardoni, S.L. Cacciatori, B.L. Cerchiai and A. Scotti, Mapping the geometry of the $E_{6}$ group, J. Math. Phys. 49 (2008), 012107, arXiv:0710.0356 [math-ph].

[36] G. Bossard, Y. Michel and B. Pioline, Extremal Black Holes, Nilpotent Orbits and the True Fake Superpotential, J. High Energy Phys. 01 (2010), 038; arXiv:0908.1742 [hep-th]. 
[37] B. de Wit, F. Vanderseypen and A. Van Proeyen, Symmetry structure of special geometries, Nucl. Phys. B 400 (1993), 463; arXiv: hep-th/9210068.

[38] S. Ferrara, E.G. Gimon and R. Kallosh, Magic supergravities, $\mathcal{N}=$ 8 and black hole composites, Phys. Rev. D 74 (2006), 125018; arXiv: hep-th/0606211.

[39] E. Cremmer and B. Julia, The $\mathcal{N}=8$ supergravity theory. 1. The Lagrangian, Phys. Lett. B 80 (1978), 48.

E. Cremmer and B. Julia, The $S O(8)$ supergravity, Nucl. Phys. B 159 (1979), 141.

[40] C. Hull and P.K. Townsend, Unity of superstring dualities, Nucl. Phys. B 438 (1995), 109; arXiv:hep-th/9410167.

[41] B. de Wit and A. Van Proeyen, Special geometry, cubic polynomials and homogeneous quaternionic spaces, Commun. Math. Phys. 149 (1992), 307; arXiv:hep-th/9112027.

[42] L. Andrianopoli, S. Ferrara, A. Marrani and M. Trigiante, Non-BPS attractors in 5d and 6d extended supergravity, Nucl. Phys. B 795 (2008), 428; arXiv:0709.3488 [hep-th].

[43] S. Ferrara and J.M. Maldacena, Branes, central charges and U duality invariant BPS conditions, Class. Quant. Grav. 15 (1998), 749; arXiv:hep-th/9706097.

[44] S. Ferrara and M. Günaydin, Orbits and attractors for $\mathcal{N}=2$ MaxwellEinstein supergravity theories in five dimensions, Nucl. Phys. B 759 (2006), 1; arXiv:hep-th/0606108.

[45] L. Borsten, D. Dahanayake, M.J. Duff, S. Ferrara, A. Marrani and W. Rubens, Observations on integral and continuous $U$-duality orbits in $\mathcal{N}=8$ supergravity, Class. Quant. Grav. 27 (2010), 185003; arXiv: 1002.4223 [hep-th].

[46] A. Strominger, Special geometry, Commun. Math. Phys. 133 (1990), 163.

[47] L. Andrianopoli, M. Bertolini, A. Ceresole, R. D'Auria, S. Ferrara, P. Fré and T. Magri, $\mathcal{N}=2$ Supergravity and $\mathcal{N}=2$ superYang-Mills theory on general scalar manifolds: symplectic covariance, Gaugings and the momentum map, J. Geom. Phys. 23 (1997), 111; arXiv: hep-th/9605032.

[48] L. Andrianopoli, R. D'Auria, S. Ferrara and M.A. Lledó, Gauging of flat groups in four dimensional supergravity, J. High Energy Phys. 07 (2002), 010; arXiv:hep-th/0203206. 
[49] A. Ceresole, S. Ferrara and A. Marrani, 4d/5d correspondence for the black hole potential and its critical points, Class. Quant. Grav. 24 (2007), 5651; arXiv:0707.0964 [hep-th].

[50] A. Ceresole, S. Ferrara and A. Marrani, Small $\mathcal{N}=2$ Extremal black holes in special geometry, Phys. Lett. B 693 (2010), 366; arXiv: 1006. 2007 [hep-th].

[51] S. Bellucci, A. Marrani and R. Roychowdhury, On quantum special Kähler geometry, Int. J. Mod. Phys. A 25 (2010), 1891; arXiv:0910. 4249 [hep-th].

[52] J. M. Landsberg and L. Manivel, A universal dimension formula for complex simple lie algebras, Adv. in Math. 201 (2004), 379; arXiv:math/0401296 [math.RT].

[53] M.J. Duff, J.T. Liu and J. Rahmfeld, Four-dimensional string-stringstring triality, Nucl. Phys. B 459 (1996), 125; arXiv:hep-th/9508094. K. Behrndt, R. Kallosh, J. Rahmfeld, M. Shmakova and W.K. Wong, STU black holes and string triality, Phys. Rev. D 54 (1996), 6293; arXiv:hep-th/9608059.

[54] M.K. Gaillard and B. Zumino, Duality rotations for interacting fields, Nucl. Phys. B 193 (1981), 221.

[55] B. de Wit and H. Nicolai, $\mathcal{N}=8$ Supergravity, Nucl. Phys. B 208 (1982), 323.

[56] A. Ceresole, R. D'Auria and S. Ferrara, The symplectic structure of $\mathcal{N}=2$ supergravity and its central extension, Nucl. Proc. Suppl. 46 (1996), 67; arXiv: hep-th/9509160.

[57] A. Ceresole, R. D'Auria, S. Ferrara and A. Van Proeyen, Duality transformations in supersymmetric Yang-Mills theories coupled to supergravity, Nucl. Phys. B 444 (1995), 92; arXiv: hep-th/9502072.

[58] E. Bergshoeff, W. Chemissany, A. Ploegh, M. Trigiante and T. Van Riet, Generating geodesic flows and supergravity solutions, Nucl. Phys. B 812 (2009), 343; arXiv:0806.2310 [hep-th].

[59] R. Gilmore, Lie Groups, Lie Algebras, and some of their applications, Dover, New York, 2006.

[60] S. Bellucci, S. Ferrara, A. Shcherbakov and A. Yeranyan, Attractors and first order formalism in five dimensions revisited, Phys. Rev. D 83 (2011), 065003; arXiv:1010.3516 [hep-th].

[61] A. Marrani, E. Orazi and F. Riccioni, Exceptional reductions, J. Phys. A 44 (2011), 155207; arXiv: 1012.5797 [hep-th] .

[62] J.C. Baez, The octonions, Bull. Amer. Math. Soc. 39 (2002), 145; arXiv:math/0105155 [math-ra]. 
[63] W. Fulton and J. Harris, Representation theory, Springer Graduate Texts in Mathematics, Springer, New York, 1991.

[64] R.S. Garibaldi, Groups of type $E_{7}$ over arbitrary fields, Commun. in Algebra 29 (2001), 2689; arXiv:math/9811056 [math.AG].

[65] S. Bellucci, S. Ferrara, M. Günaydin and A. Marrani, SAM lectures on extremal black holes in $d=4$ extended supergravity, Springer Proc. Phys. 134 (2010), 1; arXiv:0905.3739 [hep-th]. M. Günaydin, Lectures on Spectrum Generating Symmetries and U-Duality in Supergravity, Extremal Black Holes, Quantum Attractors and Harmonic Superspace, arXiv:0908.0374 [hep-th].

[66] F.W. Helenius, Freudenthal triple systems by root system methods, arXiv:1005.1275 [math.RT].

[67] H. Freudenthal, Sur le groupe exceptionnel $E_{7}$, Nederl. Akad. Wetensch. Proc. Ser. A. 56 = Indagationes Math., (1953), 15.

[68] E. Cartan, Euvres complètes (Editions du Centre National de la Recherche Scientifique, Paris, 1984).

[69] D.Z. Freedman, P. van Nieuwenhuizen and S. Ferrara, Progress toward a theory of supergravity, Phys. Rev. D 13 (1976), 3214.

[70] S. Ferrara, C. Savoy and B. Zumino, General massive multiplets in extended supersymmetry, Nucl. Phys. B 121 (1977), 393.

[71] P. Jordan, J. Von Neumann and E. Wigner, On an algebraic generalization of the quantum mechanical formalism, Ann. Math. 35 (1934), 29.

[72] L. Andrianopoli, R. D'Auria and S. Ferrara, U Invariants, black hole entropy and fixed scalars, Phys. Lett. B 403 (1997), 12; arXiv: hep-th/9703156.

S. Ferrara, A. Marrani and A. Gnecchi, $d=4$ attractors, effective horizon radius and fake supergravity, Phys. Rev. D 78 (2008), 065003; arXiv:0806.3196 [hep-th].

D. Roest and H. Samtleben, Twin supergravities, Class. Quant. Grav. 26 (2009), 155001; arXiv:0904.1344 [hep-th].

M.J. Duff and S. Ferrara, Generalized mirror symmetry and trace anomalies, Class. Quant. Grav. 28 (2011), 065005; arXiv:1009.4439 [hep-th].

[73] S. Ferrara and A. Marrani, Symmetric Spaces in Supergravity, in: "Symmetry in Mathematics and Physics" (D. Babbitt, V. Vyjayanthi and R. Fioresi Eds.), Contemporary Mathematics 490, American Mathematical Society, Providence 2009, arXiv:0808.3567 [hep-th].

[74] E. Calabi and E. Vesentini, On compact, locally symmetric Kähler manifolds, Ann. Math. 71 (1960), 472. 
[75] S. Helgason, Differential geometry, Lie groups and symmetric spaces, Academic Press, New York, 1978.

[76] E. Cremmer and A. Van Proeyen, Classification of Kähler manifolds in $\mathcal{N}=2$ vector multiplet supergravity couplings, Class. Quant. Grav. 2 (1985), 445.

[77] B.L. Cerchiai, S. Ferrara, A. Marrani and B. Zumino, Charge orbits of extremal black holes in five dimensional supergravity, Phys. Rev. D 82 (2010), 085010; arXiv:1006.3101 [hep-th].

[78] R. Mkrtchyan, A. Nersessian and V. Yeghikyan, Hopf maps and Wigner's little groups, Mod. Phys. Lett. A 26 (2011), 1393; arXiv:1008.2589 [hep-th].

[79] J.C. Baez and J. Huerta, Division algebras and supersymmetry I., arXiv:0909.0551 [hep-th].

J.C. Baez and J. Huerta, Division algebras and supersymmetry II., arXiv:1003.3436 [hep-th].

J. Huerta, Division algebras and supersymmetry III., arXiv:1109.3574 [hep-th].

[80] P. Fré, A.S. Sorin and M. Trigiante, Integrability of supergravity black holes and new tensor classifiers of regular and nilpotent orbits, arXiv:1103.0848 [hep-th].

P. Fré, A.S. Sorin and M. Trigiante, Black hole nilpotent orbits and tits satake universality classes, arXiv:1107.5986 [hep-th]. 